\title{
Spatially resolving the inhomogeneous structure of the dynamical atmosphere of Betelgeuse with VLTI/AMBER ${ }^{\star}$
}

\author{
K. Ohnaka ${ }^{1}$, K.-H. Hofmann ${ }^{1}$, M. Benisty ${ }^{2}$, A. Chelli ${ }^{3}$, T. Driebe ${ }^{1}$, F. Millour ${ }^{1,4}$, R. Petrov ${ }^{4}$, D. Schertl ${ }^{1}$, Ph. Stee ${ }^{5}$, \\ F. Vakili ${ }^{4}$, and G. Weigelt ${ }^{1}$
}

1 Max-Planck-Institut für Radioastronomie, Auf dem Hügel 69, 53121 Bonn, Germany

e-mail: kohnaka@mpifr-bonn.mpg.de

2 INAF - Osservatorio Astrofisico di Arcetri, Instituto Nazionale di Astrofisica, Largo E. Fermi 5, 50125 Firenze, Italy

3 Laboratoire d'Astrophysique de Grenoble, UMR 5571, Université Joseph Fourier/CNRS, BP 53, 38041 Grenoble Cedex 9, France

${ }^{4}$ Lab. H. Fizeau, CNRS UMR 6525, Univ. de Nice-Sophia Antipolis, Obs. de la Côte d'Azur, Parc Valrose, 06108 Nice, France

5 Lab. H. Fizeau, CNRS UMR 6525, Univ. de Nice-Sophia Antipolis, Obs. de la Côte d'Azur, Avenue Copernic, 06130 Grasse, France

Received 1 April 2009 / Accepted 22 June 2009

\section{ABSTRACT}

Aims. We present spatially resolved, high-spectral resolution $K$-band observations of the red supergiant Betelgeuse ( $\alpha$ Ori) using AMBER at the Very Large Telescope Interferometer (VLTI). Our aim is to probe inhomogeneous structures in the dynamical atmosphere of Betelgeuse.

Methods. Betelgeuse was observed in the wavelength range between 2.28 and $2.31 \mu \mathrm{m}$ with VLTI/AMBER using baselines of 16 , 32, and $48 \mathrm{~m}$. The spectral resolutions of 4800-12000 allow us to study inhomogeneities seen in the individual CO first overtone lines.

Results. Spectrally dispersed interferograms have been successfully obtained in the second, third, and fifth lobes, which represents the highest spatial resolution ( 9 mas) achieved for Betelgeuse. This corresponds to five resolution elements over its stellar disk. The AMBER visibilities and closure phases in the $K$-band continuum can be reasonably fitted by a uniform disk with a diameter of $43.19 \pm 0.03$ mas or a limb-darkening disk with $43.56 \pm 0.06$ mas and a limb-darkening parameter of $(1.2 \pm 0.07) \times 10^{-1}$. These AMBER data and the previous $K$-band interferometric data taken at various epochs suggest that Betelgeuse seen in the $K$-band continuum shows much smaller deviations from the above uniform disk or limb-darkened disk than predicted by recent 3-D convection simulations for red supergiants. On the other hand, our AMBER data in the CO lines reveal salient inhomogeneous structures. The visibilities and phases (closure phases, as well as differential phases representing asymmetry in lines with respect to the continuum) measured within the $\mathrm{CO}$ lines show that the blue and red wings originate in spatially distinct regions over the stellar disk, indicating an inhomogeneous velocity field that makes the star appear different in the blue and red wings. Our AMBER data in the CO lines can be roughly explained by a simple model, in which a patch of CO gas is moving outward or inward with velocities of $10-15 \mathrm{~km} \mathrm{~s}^{-1}$, while the $\mathrm{CO}$ gas in the remaining region in the atmosphere is moving in the opposite direction at the same velocities. Also, the AMBER data are consistent with the presence of warm molecular layers (so-called MOLsphere) extending to $\sim 1.4-1.5 R_{\star}$ with a CO column density of $\sim 1 \times 10^{20} \mathrm{~cm}^{-2}$.

Conclusions. Our AMBER observations of Betelgeuse are the first spatially resolved study of the so-called macroturbulence in a stellar atmosphere (photosphere and possibly MOLsphere as well) other than the Sun. The spatially resolved CO gas motion is likely to be related to convective motion in the upper atmosphere or intermittent mass ejections in clumps or arcs.

Key words. infrared: stars - techniques: interferometric - stars: supergiants - stars: late-type - stars: atmospheres stars: individual: Betelgeuse

\section{Introduction}

Red supergiants (RSGs) experience slow, intensive mass loss up to $10^{-4} M_{\odot} \mathrm{yr}^{-1}$. Despite its importance not only in stellar evolution but also in the chemical enrichment of the interstellar matter, the mass loss mechanism in RSGs is not well understood. While radiation pressure on dust grains is often considered to be the driving mechanism of mass loss in cool evolved stars, it is not clear where and how dust forms in RSGs and how mass outflows are initiated. Alternative scenarios include Alfvén-wave-driven

* Based on AMBER and VINCI observations made with the Very Large Telescope Interferometer of the European Southern Observatory. Program IDs: 080.D-0236 (AMBER Guaranteed Time Observation), 60.A-9054A, and 60.A-9222A. winds (Airapetian et al. 2000; Schröder \& Cuntz 2005, 2007), a combination of Alfvén waves and the wave damping due to dust (Vidotto \& Janteco-Pereira 2006), and convective turbulence combined with radiation pressure on molecules (Josselin \& Plez 2007).

The atmosphere of RSGs exhibits complicated structures. In the lower photosphere, vigorous convective motion is expected with the convective cell size possibly comparable to the stellar radius (Schwarzschild 1975; Freytag et al. 2002). The photometric variabilities, as well as the variations in the radial velocities of the metal lines in the visible, can be interpreted as caused by such giant convective cells (Kiss et al. 2006; Gray 2008). Extended chromospheres exist in the outer region. For example, the UV observations of the M supergiant Betelgeuse ( $\alpha$ Ori) with the Hubble Space Telescope revealed that the hot 
( 6000-8000 K) chromospheric plasma is more than twice as extended as the photosphere measured in the near-IR with a bright feature (Gilliland \& Dupree 1996; Uitenbroek et al. 1998). The image in the $\mathrm{H} \alpha$ line is even more extended, 4-5 times as large as the photosphere (Hebden et al. 1987), consistent with the extended chromosphere. However, radio continuum observations of Betelgeuse with the Very Large Array (VLA) suggest that much cooler $(\sim 1000-3000 \mathrm{~K})$ gas extends to several stellar radii, showing an irregular structure (Lim et al. 1998). The nonspherical shape of the outer atmosphere of Betelgeuse was also detected by mid-IR interferometric observations by Tatebe et al. (2007). Furthermore, the narrow-slit spectroscopy of Betelgeuse in the $10 \mu \mathrm{m}$ region by Verhoelst et al. (2006) reveals that silicate dust forms only at large distances from the star $\left(\gtrsim 20 R_{\star}\right)$ and that $\mathrm{Al}_{2} \mathrm{O}_{3}$ may form as close as $\sim 2 R_{\star}$. This means that the hot chromospheric plasma, cooler gas, and $\mathrm{Al}_{2} \mathrm{O}_{3}$ dust may coexist within several stellar radii from the star, but the cooler component is much more abundant compared to the chromospheric gas, because it dominates the radio emission.

The presence of cool gas in the outer atmosphere of RSGs is consistent with dense molecular layers existing close to the star, the so-called "MOLsphere", which was proposed by Tsuji (2000a, 2000b) to explain the IR spectra of the early M supergiants $\alpha$ Ori and $\mu$ Cep. While these stars were deemed too hot for $\mathrm{H}_{2} \mathrm{O}$ to form, he showed that dense $\mathrm{H}_{2} \mathrm{O}$ gas with column densities on the order of $10^{20} \mathrm{~cm}^{-2}$ and temperatures of $1500-2000 \mathrm{~K}$ at $\sim 1.3-2.0 R_{\star}$ can explain the spectral features at 2.7 and $6 \mu \mathrm{m}$, which cannot be reproduced by nongray hydrostatic photospheric models. Near- and mid-IR interferometric studies also lend support to the MOLsphere toward RSGs (e.g., Perrin et al. 2004, 2005, 2007; Ohnaka 2004a; Tsuji 2006), although the current, crude MOLsphere models cannot reproduce the $\mathrm{H}_{2} \mathrm{O}$ absorption lines observed at $12 \mu \mathrm{m}$ (Ryde et al. 2006a,b). On the other hand, Verhoelst et al. (2009) argue against such dense molecular gas being in the outer atmosphere of RSGs. They instead propose that the 2.7 and $6 \mu \mathrm{m}$ features in RSGs can be explained by a continuous (i.e. featureless) dust opacity source such as amorphous carbon and metallic iron, although it is not clear whether such grain species indeed form in oxygen-rich environments.

A better understanding of the inhomogeneous structure of the outer atmosphere of RSGs is a key to unraveling the massloss mechanism in these stars. Inhomogeneities over the stellar surface were detected by high spatial resolution imaging of a few nearby RSGs. The high-resolution images of Betelgeuse at $0.7-1.25 \mu \mathrm{m}$ with spatial resolutions of down to 30 mas (the stellar angular size is $\sim 50$ mas at these wavelengths) show the wavelength-dependent appearance of asymmetric structures (Burns et al. 1997; Tuthill et al. 1997; Young et al. 2000). However, their origin is by no means clear. They may be related to large convective cells predicted to be present in cool luminous stars (Schwarzschild 1975; Freytag et al. 2002) or alternatively to thermal instability taking place in the outer atmosphere. For example, the magnetohydrodynamical simulation of Suzuki (2007) for red giant branch (RGB) stars, which are much less luminous than RSGs, shows that thermal instability leads to "structured" stellar winds with many bubbles of hot gas $\left(\sim 10^{5} \mathrm{~K}\right)$ embedded in cool winds $\left(\sim 1-5 \times 10^{3} \mathrm{~K}\right)$. For cooler RSGs, the formation of molecules may also promote such thermal instability, and in particular, CO is an important coolant in the atmosphere of late-type stars (Cuntz \& Muchmore 1994).

To glean clues to the origin of the inhomogeneities and the mass-loss mechanism in RSGs, high spatial resolution observations in IR molecular lines are very effective. The high spectral resolution $(\lambda / \Delta \lambda=12000)$ of the near-IR interferometric instrument AMBER (Astronomical Multi-BEam combineR) at VLTI allows us to resolve the $\mathrm{CO}$ first overtone lines and to spatially resolve inhomogeneous structures within each $\mathrm{CO}$ line. In this paper, we present high-spectral and high-spatial resolution $K$-band AMBER observations of the prototypical RSG Betelgeuse (M1-2Ia-Ibe).

\section{Observations}

\section{1. $A M B E R$}

AMBER (Petrov et al. 2007) operates in the $J, H$, and $K$ bands with spectral resolutions of 35,1500 , and 12000 , combining three $8.2 \mathrm{~m}$ Unit Telescopes (UTs) or $1.8 \mathrm{~m}$ Auxiliary Telescopes (ATs). AMBER is a spectro-interferometric instrument that records spectrally dispersed fringes on the detector. With the maximum baseline length of $130 \mathrm{~m}$ currently available at the VLTI, spatially resolved spectroscopy with an angular resolution of down to 2 mas is possible with AMBER, which enables us to study the wavelength dependence of the size and shape of the object. AMBER observations with three telescopes allow us to measure three visibilities and three differential phases (DPs), as well as one closure phase (CP). Visibility is the amplitude of the Fourier transform (complex function) of the object's intensity distribution in the plane of the sky and contains information about the size and shape of the object. On the other hand, the phase of the Fourier transform (also called "Fourier phase" to avoid confusion) contains information about the object's deviation from point symmetry. While the atmospheric turbulence prevents us from measuring the phase directly, AMBER measures two observables (DP and CP) that are related to the object's phase. DP approximately represents the object's phase in a spectral feature measured with respect to the continuum ${ }^{1}$. Therefore, non-zero DPs mean a photocenter shift of the spectral feature-forming region with respect to the continuum forming region. The $\mathrm{CP}$ is the sum of phases around a closed triangle of baselines (i.e., $\varphi_{12}+\varphi_{23}+\varphi_{31}$ ) and not affected by the atmospheric turbulence. For point-symmetric objects, CP is always zero or $\pi$. Non-zero and non- $\pi$ CPs, whether in the continuum or in some spectral features, indicate the asymmetry of the object. Two-telescope AMBER observations provide only one visibility and one DP. (CP cannot be measured with two telescopes.)

Betelgeuse was observed on 2008 January 08 with AMBER using three ATs in the E0-G0-H0 array configuration with 16-32-48 $\mathrm{m}$ baselines (AMBER Guaranteed Time Observation, Program ID: 080.D-0236A, P.I.: K. Ohnaka). Since the E0-G0$\mathrm{H} 0$ configuration is a linear array lying at $+71^{\circ}$ from North $\left(+90^{\circ}=\right.$ East $)$ on the ground, the position angles of the three projected baseline vectors are the same. We used the $K$-band high-resolution mode (HR_K) of AMBER with a spectral resolution of 12000 covering wavelengths from 2.28 to $2.31 \mu \mathrm{m}$. As shown below, this wavelength range was chosen to observe the strong ${ }^{12} \mathrm{C}^{16} \mathrm{O}$ (hereafter simply $\mathrm{CO}$ ) first overtone lines near the $(2,0)$ band head. The $H$-band brightness of Betelgeuse is too high for the VLTI fringe tracker FINITO. However, the extremely high brightness of Betelgeuse $(K=-4.4)$, together with the excellent weather conditions ( $0{ }^{\prime} 3-0{ }^{\prime} 4$ seeing), enabled us to detect low-contrast fringes on all three baselines without FINITO.

\footnotetext{
1 Exactly speaking, two pieces of information are lost in the derivation of DP from AMBER observations: the absolute phase offset and the linear phase gradient with respect to wavenumber.
} 
Table 1. Summary of the AMBER observations of Betelgeuse and the calibrator Sirius.

\begin{tabular}{|c|c|c|c|c|c|c|c|c|c|c|}
\hline \# & Name & Night & $\begin{array}{c}t_{\mathrm{obs}} \\
\text { (UTC) }\end{array}$ & Tel. & $\begin{array}{l}B_{\mathrm{p}} \\
(\mathrm{m})\end{array}$ & $\begin{array}{l}\text { PA } \\
\left({ }^{\circ}\right)\end{array}$ & $\begin{array}{l}\text { Seeing (") } \\
\text { (visible) }\end{array}$ & Airmass & $\begin{array}{l}\text { DIT } \\
\text { (ms) }\end{array}$ & $\begin{array}{l}\text { Number } \\
\text { of frames }\end{array}$ \\
\hline \multicolumn{11}{|c|}{ 2008, HR_K $(\lambda / \Delta \lambda=12000), 2.28-2.31 \mu \mathrm{m}$} \\
\hline 1 & Betelgeuse & Jan. 08 & $03: 52: 58$ & E0-G0-H0 & $16.00 / 31.97 / 47.96$ & 73 & 0.4 & 1.19 & 120 & 2500 \\
\hline Cal & Sirius & Jan. 08 & 04:18:54 & E0-G0-H0 & $16.00 / 31.99 / 47.99$ & 72 & 0.3 & 1.01 & 120 & 2500 \\
\hline 2 & Betelgeuse & Jan. 08 & $04: 48: 24$ & E0-G0-H0 & $15.70 / 31.37 / 47.06$ & 71 & 0.3 & 1.28 & 120 & 2500 \\
\hline \multicolumn{11}{|c|}{$2006, \mathrm{MR} \_\mathrm{K}(\lambda / \Delta \lambda=1500), 2.1-2.2 \mu \mathrm{m}$} \\
\hline 3 & Betelgeuse & Feb. 10 & $02: 45: 08$ & E0-G0 & 15.45 & 70 & 1.1 & 1.33 & 48 & 6000 \\
\hline $\mathrm{Cal}$ & Sirius & Feb. 10 & $04: 50: 22$ & E0-G0 & 12.73 & 81 & 1.1 & 1.32 & 48 & 2500 \\
\hline
\end{tabular}

Note: $B_{\mathrm{p}}$ (projected baseline length), PA (position angle of the projected baseline vector).

We also downloaded AMBER data of Betelgeuse obtained on 2006 February 10 (Program ID: 60.A-9054A) from the ESO data archive. These data were taken with two ATs in the E0-G0$16 \mathrm{~m}$ configuration using the $K$-band medium-resolution mode (MR_K) without FINITO. The wavelength range between 2.1 and $2.2 \mu \mathrm{m}$ was covered with a spectral resolution of 1500 . Since there are no strong spectral features in this wavelength region, these MR_K data approximately sample the continuum. In both runs in 2008 and 2006, Sirius ( $\alpha$ CMa, A1V, $K=$ -1.4) was observed for the calibration of the interferometric data of Betelgeuse. We adopted an angular diameter of 5.6 mas for Sirius given by Richichi \& Percheron (2005). Observations of stars with known angular diameters and no asymmetry are needed to evaluate the so-called interferometer transfer function, which represents the instrumental and atmospheric effects on visibility and phase measurements, and for calibrating interferometric data of a science target. A summary of the observations is given in Table 1.

For the reduction of the AMBER data, we used amdlib ver. $2.2^{2}$, which is based on the P2VM algorithm (Tatulli et al. 2007). We split each data set of Betelgeuse and Sirius into five or six subsets with each subset containing 500 frames and derived the visibilities, DPs, and CPs, as well as spectra, from each subset. One of the parameters in the reduction with amdlib is the frame selection criterion. For each subset, we checked for a systematic difference in the results by taking the best $20 \%, 40 \%$, $60 \%, 80 \%$, and $100 \%$ of all frames in terms of the fringe $S / N$. We found out that the visibilities obtained from the first two subsets of the Betelgeuse data \#2, and the Sirius data taken in 2008 show a significant dependence on the selection criteria (the more frames included, the lower the visibility), while the visibilities from the other subsets do not show such a dependence. Furthermore, this dependence only occurs on the $16 \mathrm{~m}$ and $48 \mathrm{~m}$ (E0-G0 and E0-H0) baselines, while the visibilities on the $32 \mathrm{~m}$ (G0-H0) baseline are not affected by the selection criterion in any subset. The vibration of the AT at the E0 station is very likely to be the cause of this problem, because it only appears on the baselines using the E0 station, and vibration always lowers visibility. Therefore, we dropped these subsets affected by the AT vibration for the derivation of the visibilities, DPs, and CPs on the $16 \mathrm{~m}$ and $48 \mathrm{~m}$ baselines, while we used all subsets for the observables on the $32 \mathrm{~m}$ baseline. For the subsets not affected by the vibration, the reduction with different selection criteria does not lead to a significant systematic difference in the results. Selecting fewer frames only results in larger errors in the final results, while including frames with very poor $S / N$

\footnotetext{
${ }^{2}$ Available at http://www.jmmc.fr/data_processing_amber. htm
}

produces spurious results at a few wavelengths. Therefore, we included $80 \%$ of all frames in the subsets not affected by the vibration. For the AMBER MR_K data, we did not find any signature of vibration, so we used all subsets with $80 \%$ of frames included. Currently, AMBER data taken in the HR_K mode are affected by the Fabry-Perot effect caused by the InfraRed Image Sensor (IRIS), which stabilizes the image motion (see Fig. 6 in Weigelt et al. 2008). The IRIS Fabry-Perot effect is seen as timedependent, high-frequency beating in the raw visibilities, DPs, and CPs of Betelgeuse and Sirius plotted as a function of wavelength. However, fortunately, it is mostly removed by dividing the data of Betelgeuse with that of Sirius, and the IRIS beating is barely discernible in the calibrated visibilities, DPs, and CPs of Betelgeuse. This is because the data of Betelgeuse and Sirius were taken close in time. In other cases, the IRIS beating degrades the final data significantly.

While the visibilities and DPs on the shortest baseline reduced from the data taken with a spectral resolution of 12000 are of sufficient quality, the visibilities and DPs on the longer baselines and CPs turned out to be noisy. Therefore, we improved the $S / N$ for these observables by binning the data (object, dark, sky, and P2VM calibration data) in the spectral direction. For the visibilities and DPs on the middle baseline, binning with a box car filter with a width of three pixels turned out to be sufficient. This results in a spectral resolution of 8000 instead of 12000 achieved with the two-pixel sampling. For the observables on the longest baseline and CPs, it was necessary to bin the data with five pixels, corresponding to a resolution of 4800 , to obtain reasonable $S / N$ s. As shown below, the individual CO lines can still be resolved with these lowered spectral resolutions.

The errors of the resulting visibilities, DPs, and CPs were estimated from the standard deviation among the results obtained from five or six subsets. The errors of the calibrated observables were computed from such errors in the data of Betelgeuse and Sirius. Since we have only one data set for Sirius, it is impossible to estimate the systematic error in the transfer function. Therefore, to account for this error source, we added a systematic error of $5 \%$ to the above errors.

In both runs in 2008 and 2006, Sirius served not only as an interferometric calibrator but also as a spectroscopic standard star. We attempted to remove telluric lines as much as possible by dividing the spectra of Betelgeuse with that of Sirius, although the difference in air mass for the 2008 data did not allow us to achieve this perfectly. The telluric lines identified in the spectrum of Sirius were used for wavelength calibration. As a template of the telluric lines, we convolved the atmospheric transmission spectra from Wallace \& Hinkle (1996) to match the resolutions of the HR_K and MR_K modes of AMBER. The uncertainty in wavelength calibration 
is $\sim 6 \times 10^{-5} \mu \mathrm{m}\left(\sim 8 \mathrm{~km} \mathrm{~s}^{-1}\right)$ for the HR_K observations and $\sim 5 \times 10^{-4} \mu \mathrm{m}\left(\sim 70 \mathrm{~km} \mathrm{~s}^{-1}\right)$ for the MR_K observations. The wavelength scale was then converted to the heliocentric frame using the IRAF ${ }^{3}$ task RVCORRECT.

\section{2. $\mathrm{VINCl}$}

To discuss temporal variations in the $K$-band visibility, we also downloaded interferometric data of Betelgeuse taken with VINCI (VLT INterferometer Commissioning Instrument) from the ESO archive (Program ID: 60.A-9222A). These data were obtained as part of the commissioning of the instrument. A detailed description of the instrument is given in Kervella et al. (2000). As summarized in Table 2, The VINCI observations of Betelgeuse occurred on eight nights between 2001 and 2003 using two $40 \mathrm{~cm}$ siderostats in the E0-G0-16 m and B3-C3-8 m configurations (both the E0-G0 and B3-C3 configurations lie at $+71^{\circ}$ from North $\left(+90^{\circ}=\right.$ East $)$ on the ground $)$. A number of calibrators were observed on these nights (6-35 calibrator measurements on each night), as listed in Table 3.

We used the VINCI data reduction software ver.3.0 4 (Kervella et al. 2004) to derive visibility. The interferometer transfer function was computed from all calibrator measurements taken during a given night, and the mean of these transfer function values was used to obtain the calibrated visibilities of the science target. The error of each calibrated visibility was derived from the statistical error in each measurement of the science target given by the reduction software and the error in the transfer function. This latter error results from the statistical error in each calibrator measurement and the standard deviation of the transfer function values obtained on the given night. The VINCI data reduction software computes visibility using two different algorithms: Fourier transform and wavelet transform. For the data presented here, the calibrated visibilities derived with two methods agree well, and we only give the results obtained with the wavelet transform in Table 2 . The errors in the calibrated visibilities are typically $1-3 \%$. Unlike AMBER, VINCI observations were made with the $K$-broadband filter covering from 2 to $2.4 \mu \mathrm{m}$. Therefore, we computed the effective wavelength using the VINCI transmission presented in Wittkowski et al. (2004) and the spectrum of Betelgeuse observed with the Stratoscope II (detector B) by Woolf et al. (1964). The resulting effective wavelength, $2.175 \mu \mathrm{m}$, was used for calculating the spatial frequency for each observation.

\section{Results}

Figure 1 shows the calibrated visibilities, DPs, and CPs observed toward Betelgeuse as a function of wavelength. The visibilities and DPs on the middle and longest baselines, as well as the CPs were derived from the binned data (spectral resolutions of 8000 and 4800), while the results on the shortest baseline were derived from the data with a spectral resolution of 12000 . The figure reveals the detection of interferometric signals even on the longest baseline $(48 \mathrm{~m})$. This marks the highest spatial resolution (9 mas) obtained for Betelgeuse, corresponding to nearly five resolution elements over its stellar disk. The visibilities, DPs,

\footnotetext{
3 IRAF is distributed by the National Optical Astronomy Observatories, which are operated by the Association of Universities for Research in Astronomy, Inc., under cooperative agreement with the National Science Foundation.

4 Available at http://www.jmmc.fr/data_processing_vinci. htm
}

Table 2. Summary of the VINCI observations of Betelgeuse.

\begin{tabular}{|c|c|c|c|c|c|}
\hline \# & $\begin{array}{l}t_{\mathrm{obs}} \\
\text { (UTC) }\end{array}$ & $\begin{array}{l}\text { Siderostat } \\
\text { stations }\end{array}$ & $\begin{array}{r}B_{\mathrm{p}} \\
(\mathrm{m})\end{array}$ & $\begin{array}{l}\text { PA } \\
\left({ }^{\circ}\right)\end{array}$ & Visibility \\
\hline \multicolumn{6}{|c|}{2001 Oct. 12} \\
\hline 1 & $08: 11: 44$ & E0-G0 & 14.71 & 74 & $0.0988 \pm 0.0021$ \\
\hline \multicolumn{6}{|c|}{2002 Dec. 06} \\
\hline 2 & $05: 10: 46$ & B3-C3 & 7.70 & 74 & $0.4875 \pm 0.0066$ \\
\hline 3 & $05: 37: 38$ & B3-C3 & 7.89 & 73 & $0.4637 \pm 0.0086$ \\
\hline \multicolumn{6}{|c|}{2003 Jan. 07} \\
\hline 4 & $02: 40: 04$ & B3-C3 & 7.50 & 74 & $0.4752 \pm 0.0082$ \\
\hline \multicolumn{6}{|c|}{ 2003 Jan. 09} \\
\hline 5 & $01: 41: 42$ & B3-C3 & 6.79 & 74 & $0.5653 \pm 0.0069$ \\
\hline 6 & 02:10:29 & B3-C3 & 7.25 & 74 & $0.5160 \pm 0.0063$ \\
\hline \multicolumn{6}{|c|}{ 2003 Jan. 13} \\
\hline 7 & $02: 02: 03$ & B3-C3 & 7.25 & 74 & $0.5128 \pm 0.0064$ \\
\hline 8 & 02:05:11 & B3-C3 & 7.32 & 74 & $0.5093 \pm 0.0063$ \\
\hline 9 & $02: 11: 44$ & B3-C3 & 7.39 & 74 & $0.5060 \pm 0.0063$ \\
\hline 10 & $02: 15: 32$ & B3-C3 & 7.44 & 74 & $0.5060 \pm 0.0063$ \\
\hline 11 & 02:19:20 & B3-C3 & 7.48 & 74 & $0.4989 \pm 0.0062$ \\
\hline 12 & $03: 23: 52$ & B3-C3 & 7.96 & 73 & $0.4503 \pm 0.0056$ \\
\hline 13 & 03:27:39 & B3-C3 & 7.97 & 73 & $0.4461 \pm 0.0055$ \\
\hline 14 & $03: 31: 28$ & B3-C3 & 7.97 & 72 & $0.4463 \pm 0.0055$ \\
\hline 15 & 04:00:31 & B3-C3 & 7.97 & 72 & $0.4546 \pm 0.0057$ \\
\hline 16 & 04:04:18 & B3-C3 & 7.96 & 71 & $0.4519 \pm 0.0057$ \\
\hline 17 & 04:08:07 & B3-C3 & 7.95 & 71 & $0.4476 \pm 0.0056$ \\
\hline 18 & $04: 33: 46$ & B3-C3 & 7.83 & 70 & $0.4614 \pm 0.0058$ \\
\hline 19 & $04: 35: 47$ & B3-C3 & 7.81 & 70 & $0.4666 \pm 0.0058$ \\
\hline 20 & $04: 39: 36$ & B3-C3 & 7.78 & 70 & $0.4719 \pm 0.0059$ \\
\hline \multicolumn{6}{|c|}{ 2003 Jan. 14} \\
\hline 21 & $00: 49: 57$ & B3-C3 & 6.04 & 73 & $0.6780 \pm 0.0068$ \\
\hline 22 & 00:54:02 & B3-C3 & 6.13 & 73 & $0.6691 \pm 0.0068$ \\
\hline 23 & 00:58:04 & B3-C3 & 6.21 & 73 & $0.6560 \pm 0.0067$ \\
\hline 24 & 01:09:58 & B3-C3 & 6.46 & 73 & $0.5993 \pm 0.0055$ \\
\hline 25 & 01:14:00 & B3-C3 & 6.53 & 74 & $0.5942 \pm 0.0054$ \\
\hline 26 & 01:18:01 & B3-C3 & 6.61 & 74 & $0.5870 \pm 0.0054$ \\
\hline 27 & $01: 27: 23$ & B3-C3 & 6.78 & 74 & $0.5779 \pm 0.0055$ \\
\hline 28 & $01: 31: 26$ & B3-C3 & 6.85 & 74 & $0.5802 \pm 0.0057$ \\
\hline 29 & $01: 41: 48$ & B3-C3 & 7.02 & 74 & $0.5478 \pm 0.0051$ \\
\hline 30 & $01: 45: 52$ & B3-C3 & 7.08 & 74 & $0.5432 \pm 0.0051$ \\
\hline 31 & 01:49:54 & B3-C3 & 7.14 & 74 & $0.5350 \pm 0.0052$ \\
\hline 32 & $02: 54: 37$ & B3-C3 & 7.84 & 73 & $0.4871 \pm 0.0065$ \\
\hline 33 & $02: 58: 40$ & B3-C3 & 7.86 & 73 & $0.4811 \pm 0.0059$ \\
\hline 34 & 03:02:44 & B3-C3 & 7.89 & 73 & $0.4940 \pm 0.0057$ \\
\hline 35 & $03: 14: 34$ & B3-C3 & 7.94 & 73 & $0.4534 \pm 0.0043$ \\
\hline 36 & 03:18:37 & B3-C3 & 7.95 & 73 & $0.4534 \pm 0.0043$ \\
\hline 37 & $03: 22: 39$ & B3-C3 & 7.96 & 73 & $0.4573 \pm 0.0044$ \\
\hline 38 & 03:34:14 & B3-C3 & 7.98 & 72 & $0.4649 \pm 0.0045$ \\
\hline 39 & $03: 38: 18$ & B3-C3 & 7.99 & 72 & $0.4582 \pm 0.0043$ \\
\hline 40 & $03: 42: 22$ & B3-C3 & 7.99 & 72 & $0.4550 \pm 0.0044$ \\
\hline 41 & $04: 20: 35$ & B3-C3 & 7.88 & 71 & $0.4530 \pm 0.0044$ \\
\hline 42 & $04: 24: 38$ & B3-C3 & 7.86 & 70 & $0.4643 \pm 0.0043$ \\
\hline 43 & $04: 28: 43$ & B3-C3 & 7.83 & 70 & $0.4688 \pm 0.0046$ \\
\hline 44 & $00: 52: 16$ & B3-C3 & 6.17 & 73 & $0.6375 \pm 0.0087$ \\
\hline 45 & $00: 56: 20$ & B3-C3 & 6.26 & 73 & $0.6444 \pm 0.0088$ \\
\hline 46 & 01:00:21 & B3-C3 & 6.34 & 73 & $0.6269 \pm 0.0086$ \\
\hline 47 & $01: 14: 12$ & B3-C3 & 6.61 & 74 & $0.6642 \pm 0.0109$ \\
\hline 48 & $01: 18: 14$ & B3-C3 & 6.68 & 74 & $0.5902 \pm 0.0080$ \\
\hline 49 & $01: 31: 23$ & B3-C3 & 6.92 & 74 & $0.5704 \pm 0.0075$ \\
\hline 50 & $01: 35: 30$ & B3-C3 & 6.98 & 74 & $0.5612 \pm 0.0074$ \\
\hline 51 & $01: 45: 14$ & B3-C3 & 7.15 & 74 & $0.5252 \pm 0.0069$ \\
\hline 52 & 01:50:55 & B3-C3 & 7.22 & 74 & $0.5228 \pm 0.0068$ \\
\hline 53 & $01: 56: 38$ & B3-C3 & 7.30 & 74 & $0.5110 \pm 0.0067$ \\
\hline 54 & 02:07:02 & B3-C3 & 7.43 & 74 & $0.4975 \pm 0.0065$ \\
\hline 55 & $02: 12: 43$ & B3-C3 & 7.51 & 74 & $0.4847 \pm 0.0063$ \\
\hline 56 & $02: 18: 24$ & B3-C3 & 7.57 & 74 & $0.4898 \pm 0.0064$ \\
\hline 57 & 03:57:19 & B3-C3 & 7.96 & 71 & $0.4525 \pm 0.0059$ \\
\hline
\end{tabular}


Table 2. continued.

\begin{tabular}{clcccl}
\hline \hline$\#$ & $\begin{array}{l}t_{\text {obs }} \\
(\mathrm{UTC})\end{array}$ & Stations & $\begin{array}{c}B_{\mathrm{p}} \\
(\mathrm{m})\end{array}$ & $\begin{array}{c}\text { PA } \\
\left({ }^{\circ}\right)\end{array}$ \\
\hline \multicolumn{5}{c}{ 2003 Jan. 15 } \\
\hline 58 & $04: 03: 01$ & B3-C3 & 7.94 & 71 & $0.4555 \pm 0.0059$ \\
59 & $04: 08: 42$ & B3-C3 & 7.91 & 71 & $0.4618 \pm 0.0060$ \\
60 & $04: 19: 11$ & B3-C3 & 7.87 & 70 & $0.4578 \pm 0.0060$ \\
61 & $04: 25: 17$ & B3-C3 & 7.83 & 70 & $0.4765 \pm 0.0063$ \\
62 & $04: 30: 58$ & B3-C3 & 7.78 & 70 & $0.4876 \pm 0.0066$ \\
\hline \multicolumn{5}{c}{ 2003 Jan. 18 } \\
\hline 63 & $01: 11: 11$ & B3-C3 & 6.76 & 74 & $0.5671 \pm 0.0106$ \\
64 & $01: 14: 20$ & B3-C3 & 6.86 & 74 & $0.5625 \pm 0.0104$ \\
65 & $01: 28: 58$ & B3-C3 & 7.14 & 74 & $0.5296 \pm 0.0097$ \\
66 & $01: 48: 44$ & B3-C3 & 7.41 & 74 & $0.5002 \pm 0.0092$ \\
67 & $02: 03: 30$ & B3-C3 & 7.60 & 74 & $0.4676 \pm 0.0086$ \\
68 & $03: 13: 00$ & B3-C3 & 7.97 & 72 & $0.4430 \pm 0.0081$ \\
69 & $03: 15: 42$ & B3-C3 & 7.98 & 72 & $0.4389 \pm 0.0081$ \\
70 & $03: 52: 06$ & B3-C3 & 7.94 & 71 & $0.4433 \pm 0.0082$ \\
71 & $03: 55: 13$ & B3-C3 & 7.93 & 71 & $0.4458 \pm 0.0083$ \\
72 & $04: 00: 32$ & B3-C3 & 7.89 & 71 & $0.4513 \pm 0.0082$ \\
73 & $04: 12: 01$ & B3-C3 & 7.81 & 70 & $0.4557 \pm 0.0083$ \\
\hline \multicolumn{5}{c}{}
\end{tabular}

and CPs derived from the two data sets mostly show the same spectral features, demonstrating that the observed features are real despite the low fringe contrast. There seems to be a difference in visibility level for the E0-G0-16 m baseline. However, given the uncertainties in the calibrated visibilities shown in the figure, this discrepancy is marginal. We note that the CPs near the $\mathrm{CO}$ band head between 2.294 and $2.296 \mu \mathrm{m}$ are much noisier - errors of $50-100^{\circ}$ - than at the other wavelengths even in the binned data.

\subsection{Continuum: $\lambda<2.293 \mu \mathrm{m}$}

The observed spectrum of Betelgeuse below $2.293 \mu \mathrm{m}$ only shows several weak atomic and molecular absorption features, as identified in Fig. 1a. There are also subtle signatures in the observed visibilities corresponding to these features. In particular, the effects of the Ti $(+\mathrm{HF})$ feature at $2.29 \mu \mathrm{m}$ can be seen in the visibilities on all three baselines and also possibly in the CPs. These weak lines form in the deep photospheric layers and can be used for testing photospheric models, which we plan in a future paper.

The spectral resolution of the HR mode of AMBER allows us to select continuum points that are not affected by these lines. Since the visibilities on the longest baseline were derived with the five-pixel binning, we also derived the visibilities on the shorter baselines and the spectra from the data binned with five pixels. Then we selected 37 continuum points in the spectra, avoiding the lines. In Fig. 2, we plot the visibilities observed at these continuum points below $2.293 \mu \mathrm{m}$ as a function of spatial frequency. Also plotted are the AMBER MR_K data between 2.1 and $2.2 \mu \mathrm{m}$, VINCI data, $K$-band data obtained at the Infrared Optical Telescope Array (IOTA) by Perrin et al. (2004), and the $K$-band measurements by Dyck et al. (1992). The visibilities derived from the AMBER MR_K data agree very well with the result obtained by Hernández \& Chelli (2007) using not only amdlib but also another algorithm based on the Fourier transform. The errors in the visibilities from the MR_K data are $\sim 6 \%$. The DP between 2.1 and $2.2 \mu \mathrm{m}$ derived from these data is zero within a measurement error of $\sim 5^{\circ}$, which is no surprise, given the absence of strong spectral features in this wavelength range. While the AMBER HR_K and MR_K data measure the
Table 3. Calibrators used for the VINCI observations of Betelgeuse.

\begin{tabular}{|c|c|c|c|}
\hline Name & Sp.Type & $d_{\mathrm{UD}}(\mathrm{mas})$ & Night \\
\hline$\varepsilon$ Peg & K2Ib & $7.70 \pm 0.24$ & 2001 Oct. 12 (4) \\
\hline \multirow[t]{2}{*}{$\beta$ Cet } & G9II-III & $5.18 \pm 0.05$ & 2001 Oct. 12 (6) \\
\hline & & & 2002 Dec. 06 (2) \\
\hline \multirow[t]{3}{*}{$\varepsilon$ Lep } & K4III & $5.90 \pm 0.06$ & 2001 Oct. 12 (3) \\
\hline & & & 2002 Dec. 06 (1) \\
\hline & & & 2003 Jan. 09 (1) \\
\hline \multirow[t]{4}{*}{ Sirius } & A1V & $5.60 \pm 0.15$ & 2001 Oct. 12 (4) \\
\hline & & & 2002 Dec. 06 (1) \\
\hline & & & 2003 Jan. 07 (1) \\
\hline & & & 2003 Jan. 18 (6) \\
\hline \multirow[t]{2}{*}{$\delta \mathrm{CMa}$} & F8Iab & $3.29 \pm 0.46$ & 2001 Oct. 12 (2) \\
\hline & & & 2003 Jan. 07 (1) \\
\hline$\pi$ Eri & M1III & $4.8 \pm 0.5$ & 2002 Dec. 06 (1) \\
\hline \multirow{2}{*}{$\tau$ Pup } & K1III & $4.38 \pm 0.07$ & 2002 Dec. 06 (1) \\
\hline & & & 2003 Jan. 09 (1) \\
\hline \multirow{2}{*}{ Procyon } & F5IV-V & $5.37 \pm 0.11$ & 2002 Dec. 06 (1) \\
\hline & & & 2003 Jan. 07 (1) \\
\hline 1 Pup & K5III & $3.8 \pm 0.4$ & 2002 Dec. 06 (1) \\
\hline$\chi$ Phe & K5III & $2.69 \pm 0.03$ & 2002 Dec. 06 (1) \\
\hline$\iota$ Cet & K1.5III & $3.28 \pm 0.04$ & 2003 Jan. 07 (1) \\
\hline 31 Ori & K5III & $3.55 \pm 0.06$ & 2003 Jan. 07 (1) \\
\hline$\theta$ CMa & K4III & $4.13 \pm 0.4$ & 2003 Jan. 07 (1) \\
\hline \multirow[t]{2}{*}{$\beta \mathrm{Cnc}$} & K4III & $4.88 \pm 0.03$ & 2003 Jan. 07 (1) \\
\hline & & & 2003 Jan. 09 (2) \\
\hline \multirow[t]{2}{*}{$\zeta$ Нyа } & G9II-III & $3.1 \pm 0.2$ & 2003 Jan. 07 (1) \\
\hline & & & 2003 Jan. 09 (1) \\
\hline \multirow[t]{3}{*}{$\alpha$ Hya } & K3II-III & $9.44 \pm 0.9$ & 2003 Jan. 07 (1) \\
\hline & & & 2003 Jan. 09 (6) \\
\hline & & & 2003 Jan. 13 (6) \\
\hline \multirow[t]{3}{*}{$\iota$ Hya } & K2.5III & $3.41 \pm 0.05$ & 2003 Jan. 07 (2) \\
\hline & & & 2003 Jan. 09 (4) \\
\hline & & & 2003 Jan. 13 (4) \\
\hline \multirow[t]{3}{*}{$\mu$ Нуа } & K2.5III & $4.69 \pm 0.5$ & 2003 Jan. 09 (3) \\
\hline & & & 2003 Jan. 13 (9) \\
\hline & & & 2003 Jan. 15 (2) \\
\hline$\gamma^{1}$ Leo & K1IIIb & $7.70 \pm 0.70$ & 2003 Jan. 09 (3) \\
\hline \multirow[t]{3}{*}{$\beta$ Ori } & B8Iab & $2.43 \pm 0.05$ & 2003 Jan. 13 (16) \\
\hline & & & 2003 Jan. 14 (22) \\
\hline & & & 2003 Jan. 15 (15) \\
\hline V337 Car & K3IIa & $5.09 \pm 0.06$ & 2003 Jan. 14 (6) \\
\hline N Vel & K5III & $6.92 \pm 0.08$ & 2003 Jan. 07 (1) \\
\hline
\end{tabular}

Note: Uniform-disk diameters $\left(d_{\text {UD }}\right)$ are taken from Richichi \& Percheron (2005). The number of the observations of the individual calibrators on each night is indicated in parenthesis.

continuum almost free of the effects of molecular/atomic features, the VINCI and IOTA data, as well as the measurements of Dyck et al. (1992), were taken with a broad band filter spanning the entire $K$ band, which includes the molecular absorption features due to $\mathrm{CO}, \mathrm{H}_{2} \mathrm{O}$, and $\mathrm{CN}$ and atomic lines. However, since the $\mathrm{CO}$ and $\mathrm{H}_{2} \mathrm{O}$ bands only appear at limited wavelengths (CO: longward of $2.3 \mu \mathrm{m}, \mathrm{H}_{2} \mathrm{O}$ : either edge of the $K$ band), and $\mathrm{CN}$ and atomic features are weak in Betelgeuse, the total $K$-band flux is dominated by the continuum. Therefore, the data taken with the $K$ broad-band filter represent the visibilities in the continuum in first approximation, and this is why they are included in the discussion below.

To derive the angular size of the object in the continuum, we fitted the observed visibilities with a uniform disk and a limbdarkened disk. Uniform-disk fitting to the AMBER continuum data in 2008 and 2006 results in $43.19 \pm 0.03$ mas (reduced $\left.\chi^{2}=4.6\right)$ and $42.69 \pm 0.01$ mas (reduced $\chi^{2}=0.01$ ), respectively, while the fitting to all the data (AMBER, IOTA, and VINCI) results in $43.16 \pm 0.03$ mas (reduced $\chi^{2}=3.3$ ). Fitting to the 

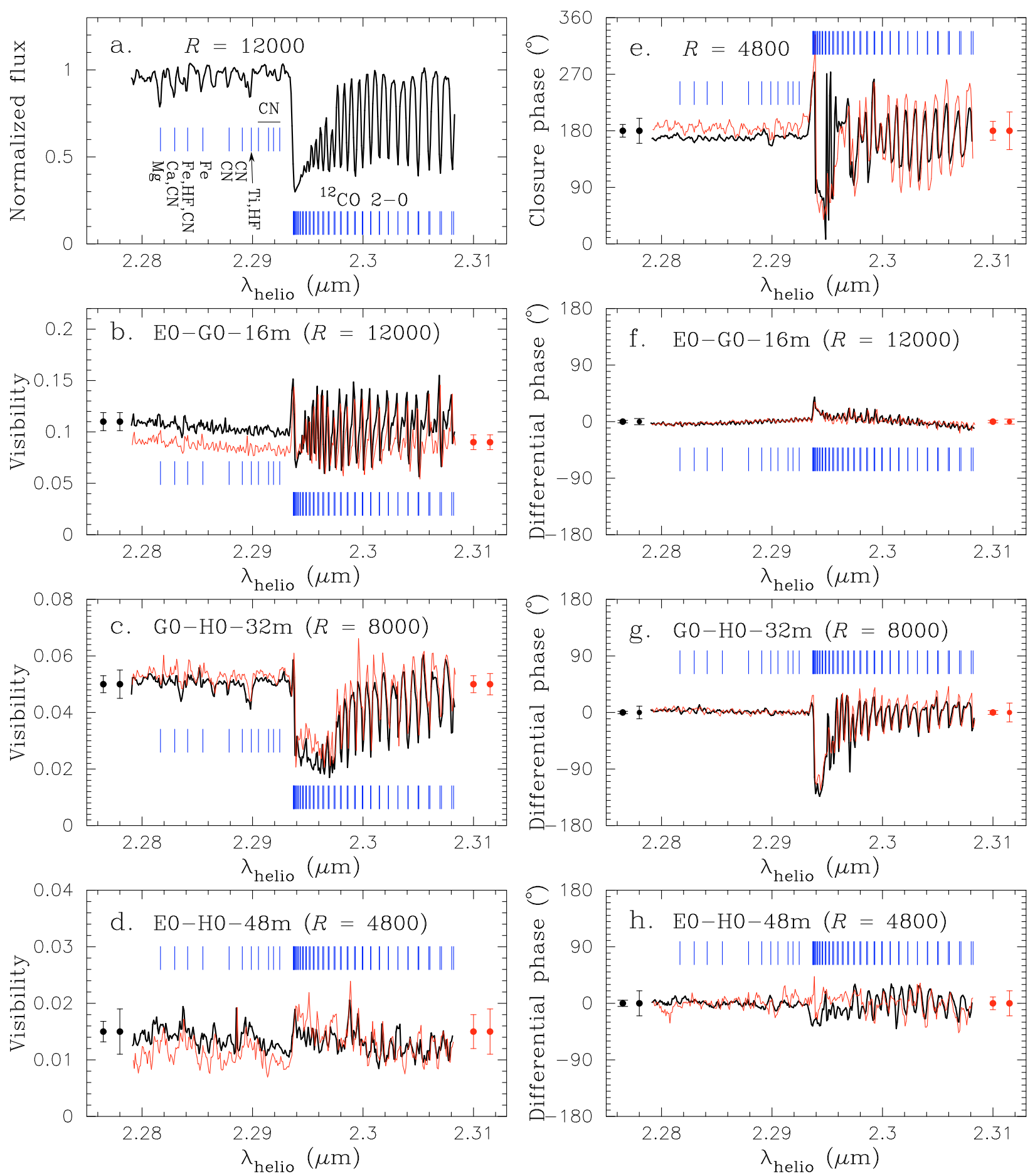

Fig. 1. AMBER observations of Betelgeuse. In all panels except for a), the black and red lines represent the data sets \#1 and \#2, respectively. The spectrum shown in the panel a) was derived from the merged data. The positions of the CO first overtone lines, as well as other atomic and molecular lines, are marked with the ticks. In the panels except for a), two error bars near the left ordinate represent the typical errors in the continuum (left) and in the CO lines (right) for the data set \#1. The errors for the data set \#2 are shown near the right ordinate in the same manner. The error in the normalized spectrum is $0.5 \%$ and $1 \%$ in the continuum and in the CO lines, respectively. The wavelength scale is in the heliocentric frame. a): Normalized flux. b)-d): Visibilities observed on the E0-G0-16 m, G0-H0-32 m, and E0-H0-48 m baselines. The visibilities on the middle and longest baselines are binned with a box car filter with widths of three and five pixels, respectively. e): Closure phases with five-pixel binning. Note that the errors in CP near the CO band head between 2.294 and $2.296 \mu \mathrm{m}$ are as large as $50-100^{\circ}$. f)-h): Differential phases observed on the E0-G0-16 m, G0-H0-32 m, and E0-H0-48 m baselines. The DPs on the middle and longest baselines are binned with three and five pixels, respectively.

AMBER HR_K data with a power-law-type limb-darkened disk (Hestroffer et al. 1997) results in a limb-darkened disk diameter of $43.56 \pm 0.06$ mas and a limb-darkening parameter of $(1.2 \pm 0.07) \times 10^{-1}\left(\right.$ reduced $\left.\chi^{2}=3.44\right)$, while fitting to all the data results in a limb-darkened disk diameter of $43.50 \pm 0.04$ mas and a limb-darkening parameter of $(9.0 \pm 0.4) \times 10^{-2}$ (reduced $\left.\chi^{2}=2.5\right)$. These results agree with the limb-darkened disk diameter and the limb-darkening parameter derived by 


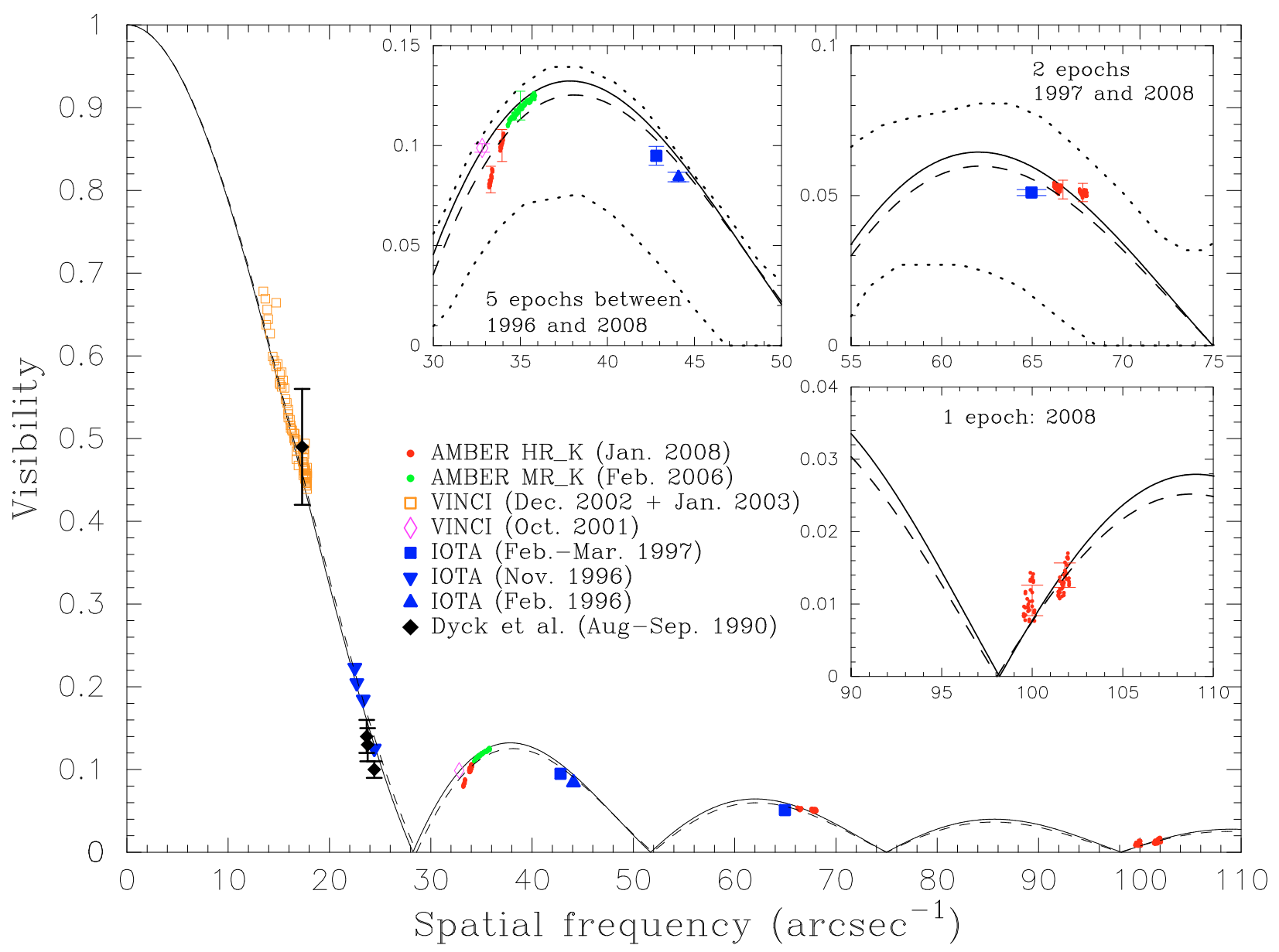

Fig. 2. $K$-band continuum/broadband visibilities of Betelgeuse plotted as a function of spatial frequency. The insets show enlarged views of the second, third, and fifth lobes. The error bars of the single AMBER data points are exemplarily shown in the insets. The errors of the VINCI and IOTA data are also shown in the insets. The solid and dashed lines represent the visibilities for a uniform disk with a diameter of 43.19 mas and for a limb-darkened disk with a diameter of 43.56 mas and a limb-darkening parameter of 0.12 (power-law-type limb-darkened disk of Hestroffer 1997), respectively. The dotted lines represent the full amplitude of the variations in the $2.22 \mu \mathrm{m}$ visibility due to time-dependent inhomogeneous structures predicted by the 3-D convection simulation of Chiavassa et al. (2007).

Perrin et al. (2004) from the $K$-broadband IOTA data. Since a uniform disk has a limb-darkening parameter of 0 , the fit suggests a small limb-darkening effect in the $K$-band continuum. Limb-darkened disk fitting to the AMBER MR_K data did not give a meaningful result, because the data are located in one narrow spatial frequency range. The fit to the AMBER HR_K data in 2008 shows that the 16,32 , and $48 \mathrm{~m}$ baselines correspond to the second, third, and fifth lobes. Although the longest baseline falls near the fourth null (i.e., between the fourth and fifth lobes), the detection of fringes in the fifth lobe is corroborated by the $180^{\circ} \mathrm{CPs}$ measured in the continuum below $2.293 \mu \mathrm{m}$ (Fig. 1e). This is because $\mathrm{CP}$ is the sum of the phases on the three baselines (E0-G0, G0-H0, and H0-E0), that is, $\mathrm{CP}=180^{\circ}$ (2nd lobe $)+0^{\circ}(3 \mathrm{rd}$ lobe $)+0^{\circ}(5$ th lobe $)=180^{\circ}$. While the above fit shows the AMBER HR_K data cannot be perfectly fitted with a uniform disk or limb-darkened disk (i.e., $\chi^{2}>1$ ), Fig. 2 suggests that the deviation from the fitted curves is not drastic at the time of the observations. This means that the spatial scale of inhomogeneities in the $K$-band continuum is even smaller than the resolution of the longest baseline ( 9 mas) and/or the contrast of the inhomogeneities is low. This picture is also supported by the result that the CPs measured in the continuum are $180^{\circ}$ within the errors, as expected from the above fit with a uniform disk or a limb-darkened disk.
The data in the second and third lobes were taken at seven different epochs as given in Fig. 2. Therefore, these data points contain information about the effects of time-dependent surface inhomogeneities on the $K$-band visibility in the continuum. Recent 3-D hydrodynamical convection simulations for RSGs by Chiavassa et al. (2007) and Ludwig \& Beckers (2008) predict significant temporal variations in visibility beyond the first null due to ever-changing inhomogeneities. For example, Chiavassa et al. (2007) present the amplitude of such variations in the $2.2 \mu \mathrm{m}$ visibility in the second and third lobes predicted for Betelgeuse, which are plotted in Fig. 2. (The model visibility at $2.1 \mu \mathrm{m}$ presented in Ludwig \& Beckers 2008 also shows similar behavior.) However, most of the observational data points in the second and third lobes are located near the upper boundary or at least in the upper half of the predicted range, instead of equally distributed above and below the center of the range. This means that the model predicts that the visibility is systematically lower than the observations. The observed data in the second lobe, where we have AMBER, VINCI, and IOTA data from five epochs, show only a modest scatter around the limbdarkened-disk fit. The data on the third lobe do not show large variations, either, although we have data from only two epochs. Therefore, the AMBER, VINCI, and IOTA observations imply that the 3-D convection simulation of Chiavassa et al. (2007) 
predicts deviations that are too large (presumably from too strong inhomogeneities) from the limb-darkening disk that fits the observed data reasonably in the $K$-band continuum.

However, given that only our AMBER HR_K data probe the very high-spatial frequency regime and the number of the observation epochs is not yet very large, we cannot conclude whether or not the visibility at these high spatial frequencies shows little deviation from a uniform-disk or limb-darkened disk all the time. Also, only our AMBER observations have measured CP, whose deviations from zero or $\pi$ would represent a clear signature of asymmetry. The visibilities of Betelgeuse observed with the $H$-broadband filter with IOTA show more deviation from a uniform disk than in the $K$ band continuum, and non-zero CPs are also detected in the third and fourth lobes (Haubois et al. 2006). While these results reveal inhomogeneous structures seen with the $H$-broadband filter (including the absorption features due to molecules such as $\mathrm{H}_{2} \mathrm{O}, \mathrm{CO}$, and $\mathrm{OH}$ ), it is not yet clear whether the amplitude of the deviation is consistent with the current convection simulation models, because the $H$-band IOTA data represent only one epoch. Further spectro-interferometric monitoring observations in the $K$ and $H$ bands at such high spatial frequencies as obtained here will enable us to study the spatial and time scale of inhomogeneities in the continuum and provide stronger observational tests for the 3-D simulations of convection in RSGs. Particularly, multi-epoch observations at the same spatial frequencies will provide a direct test for the visibility fluctuation predicted by the simulations.

Recently, Townes et al. (2009) have reported a monotonic decrease by $15 \%$ in the $11 \mu \mathrm{m}$ diameter of Betelgeuse in the past 15 years, from 1993 to 2009. Its origin is unclear. We examined a possible long-term variation in the $K$-band uniform-disk diameter in the interferometric observations in the literature and the VINCI data. The uniform-disk diameters derived from the firstlobe data in the past are 44.2 \pm 0.2 mas (Aug., Sep. 1990, Dyck et al. 1992) and 43.26 \pm 0.04 mas (Nov. 1996, Perrin et al. 2004). We fitted the VINCI data in the first lobe taken in Dec. 2002 and Jan. 2003 with a uniform disk model computed with the $K$-band transmission of VINCI and the spectrum of Betelgeuse, which were used for computing the effective wavelength. The resulting uniform-disk diameter, $42.02 \pm 0.4$ mas, agrees very well with the $42.06 \pm 0.4$ obtained by Meisner (priv. comm.) from the same VINCI data but using a different algorithm based on coherent integration (Meisner 2003). The error includes the uncertainty in the determination of the effective wavelength in VINCI observations (Meisner, priv. comm.), which we also added to the error in our uniform-disk diameter. Therefore, the $K$-broadband uniform-disk diameter shows a decrease of $5 \pm 2 \%$ from 1990 to 2003 , much less pronounced than at $11 \mu \mathrm{m}$. The continuum uniform-disk diameters of 42.69 and 43.19 mas obtained from the AMBER data taken in 2006 and 2008 might indicate an increase in the angular size after 2003. However, these diameters obtained from the data beyond the first null can be affected by inhomogeneities (although not very strong), which makes the apparent increase in the angular size inconclusive.

\section{2. $C O$ first overtone lines: $\lambda>2.293 \mu \mathrm{m}$}

Figure 1 reveals salient signatures of the $\mathrm{CO}$ first overtone lines in the visibilities, DPs, and CPs longward of $2.293 \mu \mathrm{m}$. Figure 3 shows the enlarged views between 2.299 and $2.306 \mu$ m (only the data set \#1 is plotted for visual clarity), where the observed spectrum and the line positions are overplotted in each panel to show the shape of the visibilities, DPs, and CPs within the CO lines. Each absorption feature consists of two transitions with high and
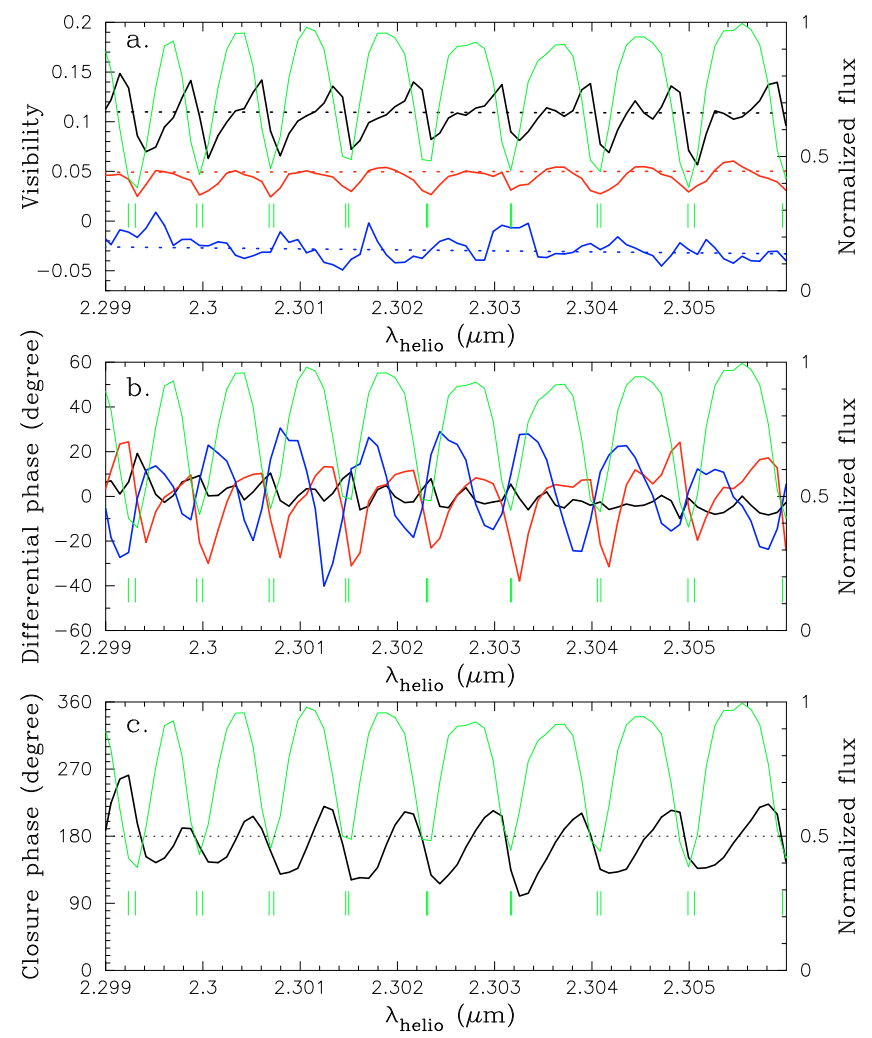

Fig. 3. Visibilities a), differential phases b), and closure phase c) observed in the $\mathrm{CO}$ first overtone lines toward Betelgeuse. In the panels a) and b), the black, red, and blue lines represent the visibilities or differential phases observed on the E0-G0-16 m, G0-H0-32 m, and E0-H0-48 m baselines, respectively. In panel a), the visibility obtained on the longest baseline (blue) is scaled by a factor of six and shifted downward by 0.1 for the sake of visual clarity. The black, red, and blue dotted lines represent the continuum visibilities of a uniform disk with 43.19 mas for the E0-G0-16 m, G0-H0-32 m, and E0-H0-48 m baselines, respectively. In panel c), the observed CP is shown by the black solid line. The dotted line represents $\mathrm{CP}=\pi$, which is observed in the continuum. The visibilities and DPs on the middle and longest baselines were derived from the data binned with three and five pixels, respectively. The $\mathrm{CP}$ was derived from the data binned with five pixels. In each panel, the normalized flux (without binning) is overplotted in green to show the behavior of the visibilities and phases within individual $\mathrm{CO}$ lines. The positions of the CO lines are marked with the vertical ticks. The wavelength scale is in the heliocentric frame. A color version of this figure is available in the electronic edition.

low rotational quantum numbers $J$. But for simplicity, we refer to such an absorption feature with high and low $J$ as a "line" in the present work. Comparison of the observed line positions with the laboratory data results in a heliocentric velocity of $24 \mathrm{~km} \mathrm{~s}^{-1}$. This value agrees with the velocity range $17-27 \mathrm{~km} \mathrm{~s}^{-1}$ derived from the visible and IR atomic and molecular lines, as well as the mm CO lines (e.g., Brooke et al. 1974; Huggins 1987; Smith et al. 1989; Huggins et al. 1994; Ryde et al. 1999).

Figure 3 a shows the visibilities observed on the three baselines. Particularly surprising is that the visibility observed within a given CO line on the shortest baseline $(\sim 16 \mathrm{~m})$ is antisymmetric with respect to the line core (i.e., " $\sim$ "-shaped). While the visibility on the middle baseline $(\sim 32 \mathrm{~m})$ is roughly symmetric, the visibility on the longest baseline $(\sim 48 \mathrm{~m})$ is asymmetric with the peak slightly redshifted with respect to the line core in most cases. These results mean that Betelgeuse appears different in the blue and red wings of the CO lines and that the blue and red wings originate in spatially distinct regions differing in size 
and/or shape. The observed DPs and CPs show remarkable nonzero and non- $\pi$ values, as high as DP $=-130^{\circ}$ (Fig. $1 \mathrm{~g}$ ) or $\mathrm{CP}=$ $180^{\circ} \pm 90^{\circ}$ (Figs. 1e and 3c). These non-zero and non- $\pi$ DPs and $\mathrm{CPs}$ confirm that the blue and red wings of the $\mathrm{CO}$ lines originate in spatially distinct regions. On the other hand, the observed DPs and CPs are nearly zero near the line core, which means that the star appears symmetric.

One might suspect that such blue-red asymmetry in visibilities, DPs, and CPs within the CO line profiles can be explained by one $\mathrm{CO}$ absorption feature, which appears to be a single line, being a blend of two transitions with low and high $J$. If two lines with different excitation potentials form at different regions over the stellar surface, it may make the star appear different in the blue and red wings and cause the blue-red asymmetry. However, the relative positions of the high and low $J$ lines switch at $2.3032 \mu \mathrm{m}$ : shortward of this wavelength, the high $J$ lines appear blueward of the low $J$ lines, while above $2.3032 \mu \mathrm{m}$, the high $J$ lines appear redward of the low $J$ lines. Still, the same asymmetry is observed in all $\mathrm{CO}$ lines, whether shortward or longward of $2.3032 \mu \mathrm{m}$. Therefore, the observed asymmetry in the visibility, DPs, and CP cannot be explained by the blend of two transitions with high and low $J$.

Stellar rotation or spherically expanding/infalling flows are also unlikely to be the cause of the blue-red asymmetry in visibilities and phases. The projected photospheric rotational velocity of Betelgeuse is low, $v \sin i \approx 2-5 \mathrm{~km} \mathrm{~s}^{-1}$ (Uitenbroeck 1998; Harper \& Brown 2006). Moreover, the rotational axis inferred from the chromospheric emission lines lies at $\sim 65^{\circ}$ (Harper \& Brown 2006), which is very close to the position angle of the projected baselines of our AMBER observations. In this case, rotation can have no noticeable effect on visibilities and phases. In spherically expanding/infalling flows, the velocity in the line of sight changes as a function of the angular distance from the stellar disk center. Therefore, the photons at different wavelengths (=different velocities) within a line profile originate in annular regions with different sizes, which makes the star appear different in the blue and red wings. However, such spherically symmetric models obviously cannot explain the observed nonzero/non- $\pi$ DPs and CPs.

The observed blue-red asymmetry in visibility, DP, and CP may be explained by an inhomogeneous velocity field in the atmosphere, in which upwelling and downdrafting $\mathrm{CO}$ gas exist in spatially distinct regions. In spectroscopic analyses using 1-D model atmospheres, such a non-thermal velocity field is empirically incorporated as macroturbulence, which manifests itself as a broadening of spectral lines ("macro" means the spatial scale of the non-thermal gas motion is larger than the length of the unit optical depth of photons). In the next section, we examine whether the observed visibilities, DPs, and CPs can be explained by such an inhomogeneous velocity field.

\section{Modeling}

To characterize the inhomogeneous velocity field and the properties of the $\mathrm{CO}$ gas in the atmosphere of Betelgeuse, we constructed the following patchy two-layer model, in which the star is surrounded by the inner and outer CO layers. The star was assumed to be a blackbody of an effective temperature of $3600 \mathrm{~K}$ based on the value derived by Perrin et al. (2004). The inner layer represents the photosphere - the region included in 1-D photospheric models, usually spanning continuum optical depths of $\sim 10^{1}-10^{2}$ to $\sim 10^{-5}-10^{-6}$. The outer layer represents the MOLsphere. Given the dense $\mathrm{H}_{2} \mathrm{O}$ gas in the MOLsphere suggested for Betelgeuse from spectroscopic and

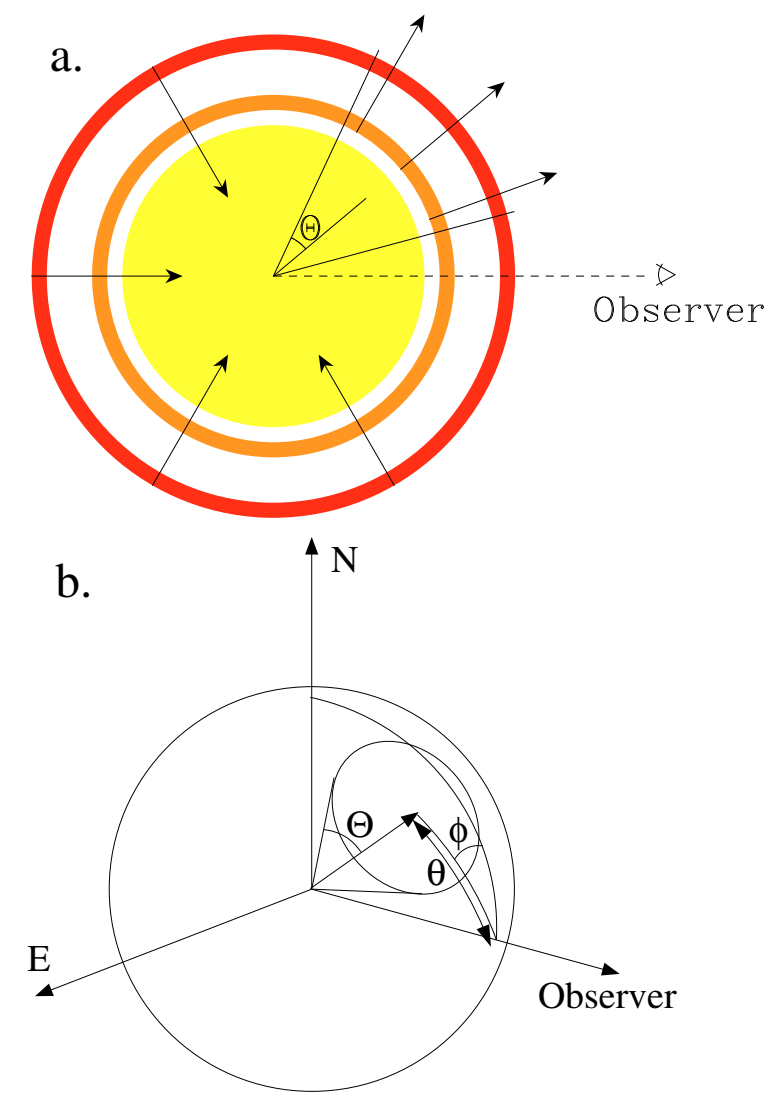

Fig. 4. Schematic view of our patchy model for Betelgeuse. a) Cross section of the model. The star is surrounded by two layers with an inhomogeneous velocity field. b) 3-D view of the model. The $\mathrm{N}$ - and E-axes define the plane of the sky. Only one layer is drawn for the sake of visual clarity. Within the cone specified by a half-opening angle of $\Theta$ and the position $(\theta, \phi)$ with respect to the observer, the CO gas is assumed to be moving radially outward (or inward), while it is moving radially inward (or outward) in the remaining region.

interferometric observations (Perrin et al. 2004, 2007; Ohnaka 2004a; Tsuji 2006), it is plausible that there is also a significant amount of $\mathrm{CO}$ in the MOLsphere.

The basic picture of our model is depicted in Fig. 4. This two-layer model is similar to the one described in Ohnaka (2004b), but we introduced two modifications. First, the geometrical thickness of each layer is assumed to be very small compared to its radius, therefore the two layers are detached from each other. This simplifies the computation of the line opacity in the presence of a velocity field as described below. Second, while the column density and temperature of each layer were assumed to be constant over the stellar surface, we incorporated the following inhomogeneous velocity field: $\mathrm{CO}$ gas is moving radially outward (or inward) with a velocity of $v_{\text {flow }}$ in one patch, while it is moving in the opposite direction at the same velocity in the remaining region. We assumed the same velocity field for two layers. As Fig. 4b illustrates, we define such a patch as a cone. Its half-opening angle $(\Theta)$ characterizes the size of the patch, while its position is specified by two angles, $\theta$ and $\phi$, which define the vector connecting the center of the patch and the center of the star.

We do not know a priori the actual number and shape of patches (if any) on the stellar surface, and the present data are insufficient to derive the inhomogeneous surface pattern uniquely. We only assume one patch in our modeling to keep the number of free parameters as small as possible. The aim of our modeling 

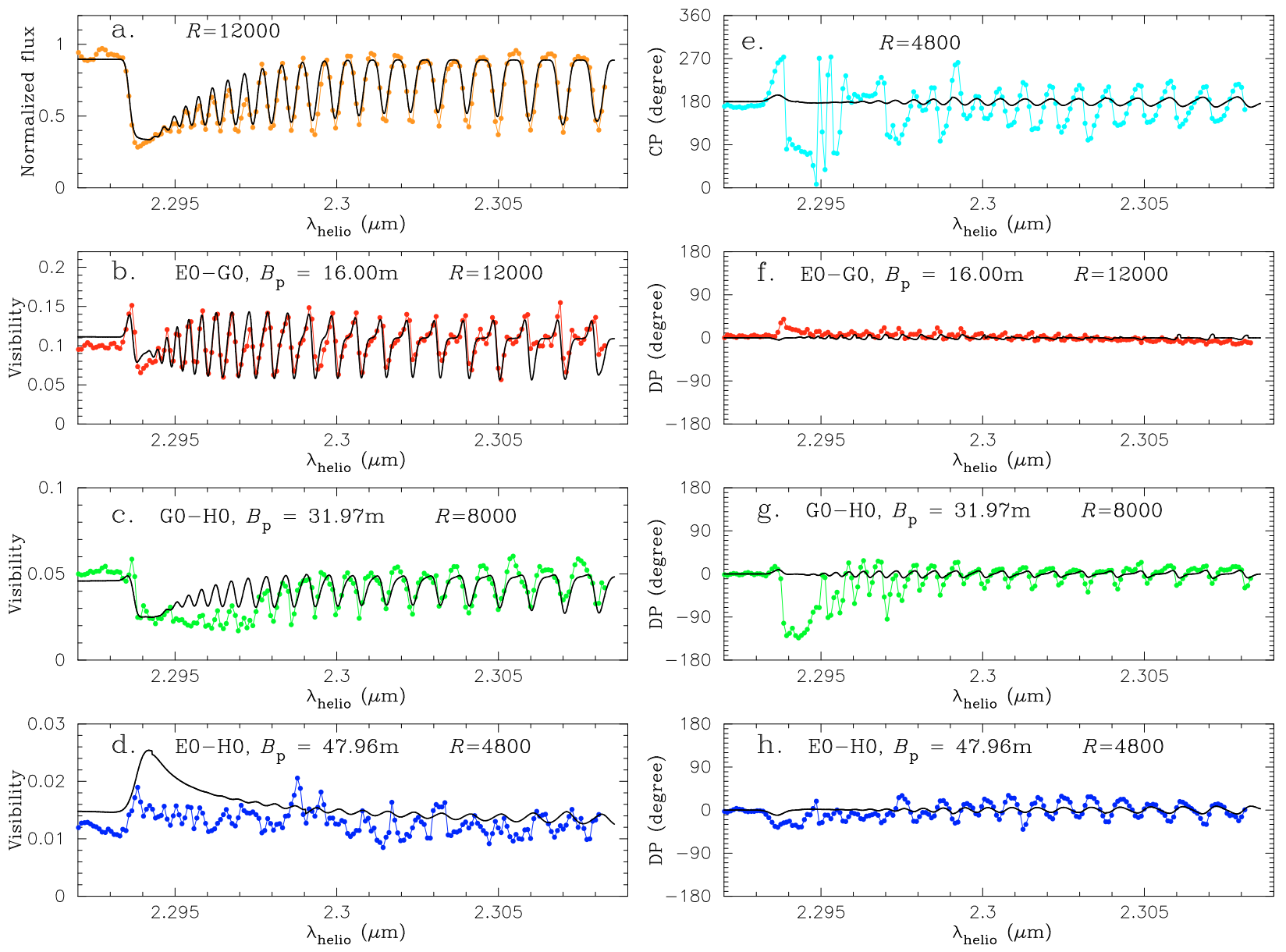

Fig. 5. Comparison between our patchy model $\left(T_{\text {in }}=2250 \mathrm{~K}, N_{\text {out }}=1 \times 10^{20} \mathrm{~cm}^{-2}, v_{\text {flow }}=10 \mathrm{~km} \mathrm{~s}^{-1}, \Theta=60^{\circ}, \theta=40^{\circ}\right.$, and $\left.\phi=10^{\circ}\right)$ and the AMBER data for Betelgeuse. In all panels, the solid lines represent the model, while the dots represent the observational data (data set \#1). a): Normalized flux. b)-d) Visibilities on the E0-G0-16 m, G0-H0-32 m, and E0-H0-48 m baselines. The observed and model visibilities on the last two baselines are binned with three and five pixels, respectively. e): Closures phase. The observed data and the model are binned with five pixels. f)-h): Differential phases on the E0-G0-16 m, G0-H0-32 m, and E0-H0-48 m baselines. The observed and model DPs on the last two baselines are binned with three and five pixels, respectively.

is not to derive the actual inhomogeneous surface structure but to see whether there is indeed a model with an inhomogeneous velocity field that can explain the observed blue-red asymmetry in visibility, DP, and $\mathrm{CP}$ within the $\mathrm{CO}$ lines.

To decrease the number of free parameters, we fixed the CO column density and radius of the inner layer as follows. A spherical photospheric model with the molecular opacities due to $\mathrm{CO}, \mathrm{TiO}, \mathrm{H}_{2} \mathrm{O}, \mathrm{OH}$, and $\mathrm{SiO}$ incorporated based on the opacity sampling (Ohnaka, in prep.) was computed using the stellar parameters of Betelgeuse given in Tsuji (2006). This photospheric model gives a CO column density of $5 \times 10^{22} \mathrm{~cm}^{-2}$, which we adopted for the inner layer. We assumed the radius of the inner CO layer to be a half of the geometrical thickness of this photospheric model $\left(\sim 0.1 R_{\star}\right)$, that is, a radius of $1.05 R_{\star}$. While this choice is ambiguous, it turned out not to affect the result significantly, as far as the radius is smaller than $\sim 1.1 R_{\star}$. It is necessary to include the microturbulence, which represents the non-thermal gas motion on a spatial scale smaller than the length of the unit optical depth of photons. The microturbulent velocity in the photosphere of Betelgeuse derived from spectroscopic analyses ranges from 4 to $6 \mathrm{~km} \mathrm{~s}^{-1}$ (Lambert et al. 1984; Tsuji et al. 1994; Tsuji 2006). We adopted a microturbulent velocity of $5 \mathrm{~km} \mathrm{~s}^{-1}$ for both the inner and outer $\mathrm{CO}$ layers in our modeling.
For the outer layer, we fixed its radius and temperature based on the previous spectroscopic and interferometric studies of the MOLsphere. The radius of the MOLsphere of Betelgeuse measured from the near- and mid-IR $\mathrm{H}_{2} \mathrm{O}$ features is $1.45 R_{\star}$ (Ohnaka 2004a), $1.3 R_{\star}$ (Tsuji 2006), and 1.31-1.43 $R_{\star}$ (Perrin et al. 2007). We tentatively adopted $1.45 R_{\star}$ for our modeling. The temperature of the $\mathrm{H}_{2} \mathrm{O}$ MOLsphere derived by the above authors ranges from 1500 to $2250 \mathrm{~K}$. A gas temperature of $1800 \mathrm{~K}$, which lies roughly in the middle of this range, was adopted for the outer CO layer. Therefore, the free parameters of our model are the temperature of the inner $\mathrm{CO}$ layer $\left(T_{\mathrm{in}}\right)$, the CO column density of the outer CO layer $\left(N_{\text {out }}\right)$, the velocity of the $\mathrm{CO}$ gas motion $\left(v_{\text {flow }}\right)$, and the position and size of the patch $(\theta, \phi$, and $\Theta)$.

The intensity distribution for this patchy model was computed at each wavelength between 2.291 and $2.309 \mu \mathrm{m}$ by performing ray tracing along a number of lines of sight (see Ohnaka 2004a,b). The only difference from these previous studies is the inclusion of the Doppler shift due to the velocity field in the calculation of the line opacity. Since each layer is geometrically thin, the velocity component along the line of sight within one layer can be approximated to be constant, which simplifies the computation. The CO line list of Goorvitch (1994) was used for calculating the line opacity. The monochromatic 2-D complex 

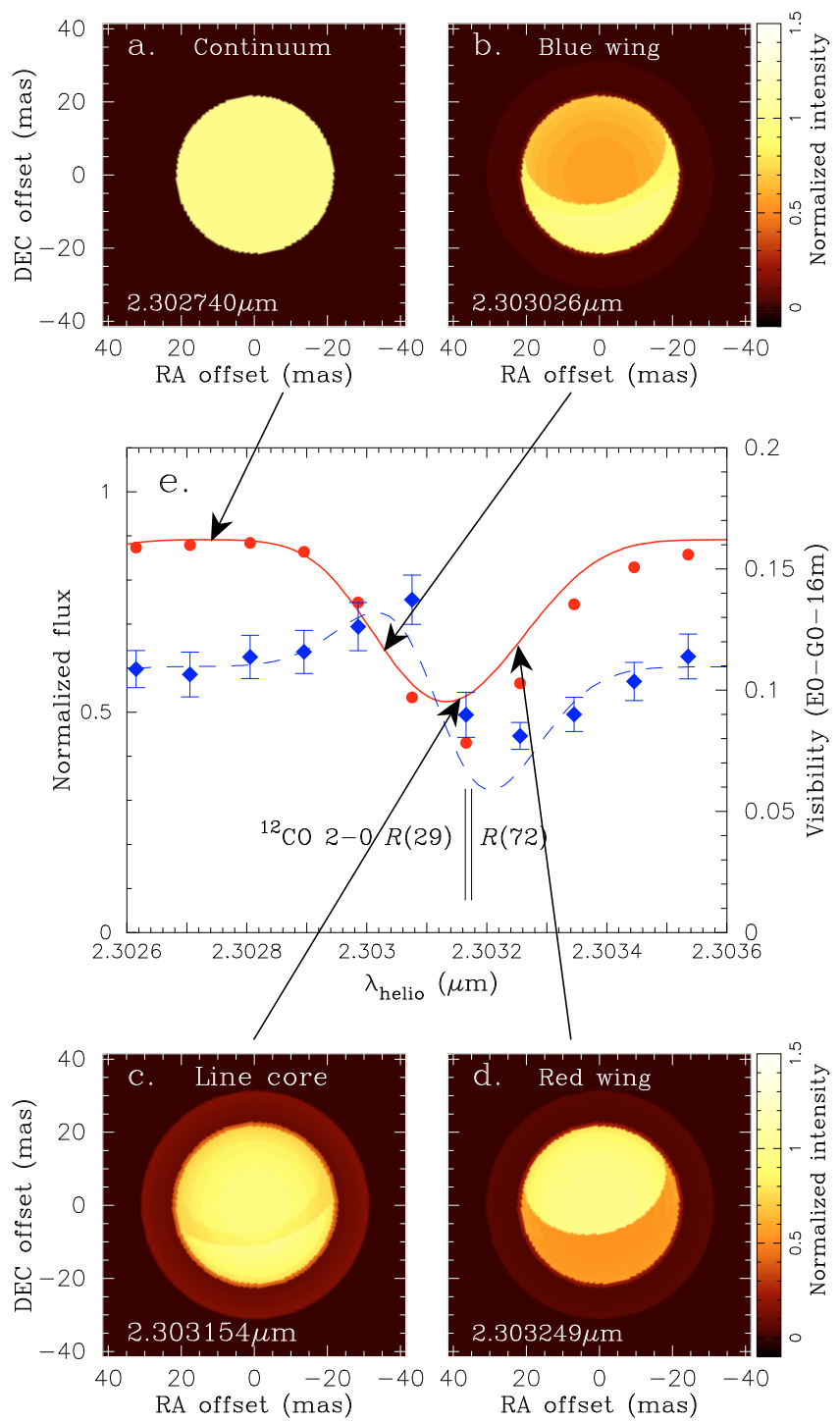

Fig. 6. Images of Betelgeuse predicted by our patchy model shown in Fig. 5 at different wavelengths in the $\mathrm{CO}$ line consisting of $R(29)$ and $R(72)$. a)-d): Model images in the continuum, blue wing, line core, and red wing as indicated in the panel e). The extended MOLsphere is slightly visible in the panel c). e): Normalized flux (left ordinate) and visibility on the shortest baseline (right ordinate). The filled circles and solid line represent the observed and model flux, respectively. The observed visibility and model prediction are shown by the filled diamonds and dashed line, respectively. The line positions are marked with the vertical ticks. A color version of this figure is available in the electronic edition.

visibility was calculated by taking the Fourier transform of the intensity and then was spectrally convolved to match the resolution of our AMBER observations (12000, 8000, and 4800). The visibility amplitude and phase were derived from this spectrally convolved 2-D complex visibility, which was scaled with the uniform-disk diameter of 43.19 mas derived from the AMBER HR_K data in the continuum. CP was obtained as the sum of Fourier phases predicted for three baselines, while DP on a given baseline was computed by taking the difference in Fourier phase between a CO line and the continuum below $2.293 \mu \mathrm{m}$. The correspondence between the sign of $\mathrm{CP}$ measured with AMBER and the positional offset in the plane of the sky is described in Kraus et al. (2009).
Figure 5 shows a model with $T_{\text {in }}=2250 \mathrm{~K}$ and $N_{\text {out }}=$ $1 \times 10^{20} \mathrm{~cm}^{-2}$, characterized by a large, upwelling spot $\left(\Theta=60^{\circ}\right.$, $v_{\text {flow }}=10 \mathrm{~km} \mathrm{~s}^{-1}$ ) covering nearly a half of the apparent stellar disk $\left(\theta=40^{\circ}, \phi=10^{\circ}\right.$, see also Figs. $6 \mathrm{~b}$ and $\left.6 \mathrm{~d}\right)$. Given the simple nature of the model, the overall agreement is reasonable, although there are still discrepancies between the model and the observed data. Particularly, the agreement is poor near the band head, which is discussed below. Also, the visibility on the longest baseline as well as the $\mathrm{CP}$ predicted by the model is too smooth compared to the observed data (note that the spectral resolution of the model matches that of the binned data). Figure 6 shows the images predicted by this model at different wavelengths within a CO line $(R(29)$ and $R(72))$. The figure illustrates that the velocity field makes the star appear different in the blue and red wings. This can cause the visibility to be anti-symmetric with respect to the line core as observed on the shortest baseline and also explains the observed asymmetric DPs and CPs. The stellar image at the line core is the sum of the blueand red-shifted components. This makes the star appear nearly centrosymmetric (Fig. 6c) and results in DPs and CPs close to zero at the line core as observed.

The agreement between the observed data and the model becomes poorer near the band head. In particular, the deviations of DP from zero predicted for the middle and longest baselines are too small compared to the observed values. The reason why the DPs predicted by the model do not show significant deviation from zero is as follows. Since the lines are very crowded near the band head, the blue (or red) wing of one line overlaps with the red (or blue) wing of the adjacent lines. This means that the appearance of the star at a given wavelength in such a crowded region is the sum of the blue- and red-shifted components as in the case of the image at the core of an isolated line. As Fig. 6c shows, the resulting image appears roughly centrosymmetric. This is the reason why the DPs predicted by the model do not show noticeable deviations from zero. A possible reason for the discrepancy near the band head is our assumption that the $\mathrm{CO}$ column density and temperature are uniform over the stellar surface. Inhomogeneities in CO column density and/or in temperature over the surface may reconcile the disagreement between the model and the observed data near the band head.

The uncertainty ranges of $T_{\text {in }}, N_{\text {out }}$, and $v_{\text {flow }}$ derived from our modeling are $2000-2500 \mathrm{~K}, 5 \times 10^{19}-2 \times 10^{20} \mathrm{~cm}^{-2}$, and $10-15 \mathrm{~km} \mathrm{~s}^{-1}$, respectively. We also computed models with $N_{\text {out }}$ set to zero (i.e., no MOLsphere), but such models cannot reproduce the visibilities observed near the band head. The uncertainties in the position and size of the patch are very large: the ranges for $\Theta, \theta$, and $\phi$ are $20^{\circ}-80^{\circ}, 20^{\circ}-80^{\circ}$, and $0^{\circ}-40^{\circ}$, respectively. However, these uncertainties in the position and size of the patch should not be taken at face value, because we assumed for simplicity that there is only one spot, which may not be the case. Also, we cannot conclude that the $\mathrm{CO}$ gas in the patch is moving upward or downward. We computed models in which the patch is moving downward and found out that there are parameter sets that are consistent with the observations. However, apart from these uncertainties in the actual surface pattern and the direction of the velocity field, our AMBER data and modeling suggest an inhomogeneous velocity field with amplitudes of $\sim 10-15 \mathrm{~km} \mathrm{~s}^{-1}$.

\section{Discussion}

The amplitude of the velocity field suggested from our modeling, $10-15 \mathrm{~km} \mathrm{~s}^{-1}$, compares favorably with the macroturbulent velocities derived in the previous spectroscopic analyses. 
Macroturbulence manifests itself as the broadening of spectral lines in observed spectra. In spectral analyses using 1-D model atmospheres, macroturbulence is usually incorporated as an additional line broadening factor with a Gaussian distribution (i.e. $\left.\propto \exp \left(-\left(\lambda / \lambda_{\text {macro }}\right)^{2}\right)\right)$. The macroturbulent velocity - either as the Gaussian dispersion $V_{\text {macro }}$ or the $F W H M=1.665 V_{\text {macro }}$ corresponding to $\lambda_{\text {macro }}-$ is derived so that synthetic spectra reproduce observed line profiles. For Betelgeuse, macroturbulent velocities as high as $20 \mathrm{~km} \mathrm{~s}^{-1}(F W H M)$ were derived from the optical lines by Lobel \& Dupree (2000) and Gray (2000), while lower values of $10-12 \mathrm{~km} \mathrm{~s}^{-1}$ were obtained from near- and midIR lines (Jennings et al. 1986; Jennings \& Sada 1998; Ryde et al. 2006a; Tsuji 2006).

Josselin \& Plez (2007) have analyzed spectral line profiles in the optical and extracted information about the velocity field in a sample of RSGs. For Betelgeuse, they find that the strong lines with lower excitation potentials of $\sim 1 \mathrm{eV}$ show blue- and red-shifted components at approximately $\pm 10 \mathrm{~km} \mathrm{~s}^{-1}$ with respect to the velocity of weak lines with excitation potentials of $\sim 3 \mathrm{eV}$. These velocities agree roughly with the $10-15 \mathrm{~km} \mathrm{~s}^{-1}$ derived from our modeling of the $\mathrm{CO}$ first overtone lines with excitation potentials of $<1 \mathrm{eV}$. Josselin \& Plez (2007) detect no periodic or regular temporal variation in the velocity of the blueand red-shifted components, which led them to interpret these two components as upward and downward convective motion.

On the other hand, it is not so obvious whether such strong convective motion can be present in the $\mathrm{CO}$ line formation layers, where the convective energy flux is small. Alternatively, it is possible that the $\mathrm{CO}$ gas motion detected by our AMBER observations may represent local mass ejections. High-resolution studies of the dusty RSG VY CMa and less massive evolved stars, such as IRC+10216 and CIT6, suggest that the mass loss is accompanied by episodic mass ejections in clumps or arcs (Humphreys et al. 2007; Smith et al. 2009; Weigelt et al. 2002; Monnier et al. 2000). While the circumstellar envelope around Betelgeuse is spherical on a large scale unlike VY CMa, inhomogeneities such as clumps and plumes have been detected (Plez \& Lambert 2002; Smith et al. 2009). The recent high-resolution images in the near-IR taken by Kervella et al. (2009) reveal a large plume extending to roughly six stellar radii in the southwestern region of the star. The long-term variability of the $\mathrm{H} \alpha$ line velocity can also be interpreted as a consequence of "intermittent failed ejections", in which material is flung out and falls back toward the star (Smith et al. 1989).

To summarize, our AMBER observations are the first spatially-resolved detection of macroturbulent gas motion in a stellar atmosphere (photosphere and possibly MOLsphere) other than the Sun ${ }^{5}$. The spatially resolved CO gas motion can represent the convective motion in the upper photosphere (and also in the MOLsphere) or the motion related to intermittent mass ejections in clumps and/or arcs.

The $(u, v)$ coverage of the present AMBER data is insufficient for deriving the actual inhomogeneous structure in the photosphere and MOLsphere. In particular, the lack of visibilities in the first lobe makes it difficult to measure the size of the MOLsphere seen in the CO lines, and we simply assumed the same radius and temperature as those derived from the near- and mid-IR $\mathrm{H}_{2} \mathrm{O}$ features. While baselines shorter than the $16 \mathrm{~m}$ currently available at the VLTI is desirable, it is also possible to observe Betelgeuse in the first lobe by taking advantage of the

5 The chromospheric gas motion toward Betelgeuse was spatially resolved with the HST observations in the UV by Gilliland \& Dupree (1996). projection effect. Such new data will be indispensable for constraining the geometrical extent of the $\mathrm{CO}$ gas in the MOLsphere of Betelgeuse.

Lastly, we estimate the number of observations needed to reconstruct an image. As a rule of thumb, the number of $(u, v)$ points should be larger than the number of filled pixels (i.e., pixels with stellar flux) in the reconstructed image (e.g., Haniff 2007). This means that we need at least five $(u, v)$ points - realistically about 10 points - to reconstruct a 1-D image with five resolution elements over the stellar disk as in our present work. Therefore, to reconstruct a $2-D$ image with $5 \times 5$ pixels, we need approximately $100(u, v)$ points, which corresponds to $\sim 33$ observations ( $\sim 3$ nights) with AMBER using three telescopes. It is crucial that these $(u, v)$ points are as uniformly distributed as possible, which will become feasible when more new VLTI configurations, particularly short baselines perpendicular to E0-G0$\mathrm{H} 0$, are opened. The above estimate is roughly consistent with the image reconstruction simulation for the VLTI 2nd generation instrument MATISSE (Hofmann et al. 2008).

\section{Concluding remarks}

We have spatially resolved the $\mathrm{CO}$ gas motion in the atmosphere of Betelgeuse with high-spectral resolution using VLTI/AMBER and successfully measured the visibilities, DPs, and CPs in the second, third, and fifth lobes, marking the highest spatial resolution ( $~ 9$ mas) achieved for Betelgeuse. The visibilities observed in the $\mathrm{CO}$ first overtone lines suggest that the blue and red wings of individual lines originate in spatially distinct regions, and the non-zero/non- $\pi$ DPs and CPs observed in the CO lines corroborate this picture. Our simple model suggests an inhomogeneous velocity field with amplitudes of $\sim 10-15 \mathrm{~km} \mathrm{~s}^{-1}$ in the atmosphere of Betelgeuse. These AMBER observations are the first to spatially resolve the so-called macroturbulence in a stellar atmosphere other than the Sun. The spatially resolved CO gas motion is likely to correspond to the convective motion in the upper photosphere (and possibly MOLsphere as well) or intermittent, failed clumpy mass ejections. Our modeling also shows that the AMBER data are consistent with the presence of the MOLsphere extending to $\sim 1.45 R_{\star}$ with a CO column density of $\sim 10^{20} \mathrm{~cm}^{-2}$ and a temperature of $1800 \mathrm{~K}$.

The visibilities and CPs observed in the continuum below $2.293 \mu \mathrm{m}$ do not show any drastic deviation from a limb-darkened disk with a diameter of 43.56 mas and a limbdarkening parameter of 0.12 at the time of the observations. Comparison of our AMBER HR_K data with the previous AMBER MR_K data, VINCI data, and IOTA measurements reveals that the recent 3-D convection simulations for Betelgeuse predict the $K$-band continuum visibility to be too low beyond the first null.

We plan to continue AMBER observations of Betelgeuse to study temporal variations in the visibilities and phases in the CO lines, as well as in the continuum. Such high-spatial resolution data will provide tight constraints on the time and spatial scale of the inhomogeneities. When more baselines, particularly short ones, become available, reconstruction of a $5 \times 5$-pixel image will be feasible with observations in $\sim 3$ nights. Highresolution imaging for various molecular lines will be essential for understanding the physical processes responsible for the inhomogeneous structures in RSGs.

Acknowledgements. We thank the ESO VLTI team in Garching and in Paranal, particularly Fredrik Rantakyrö, for supporting our AMBER observations. We also thank Jeff Meisner for his reduction of the VINCI data and discussion of the accuracy of the determination of the diameter diameters from the VINCI data. 


\section{References}

Airapetian, V. S., Ofman, L., Robinson, R. D., Carpenter, K., \& Davila, J. 2000, ApJ, 528, 965

Brooke, A. L., Lambert, D. L., \& Barnes III, T. G. 1974, PASP, 86, 419

Burns, D., Baldwin, J. E., Boysen, R. C., et al. 1997, MNRAS, 290, L11

Chiavassa, A., Plez, B., Josselin, E., \& Freytag, B. 2007, SF2A-2007, ed. J. Bouvier, A. Chalabaev, \& C. Charbonnel, 447, also available at [arXiv:astro-ph/0802.1403]

Cuntz, M., \& Muchmore, D. O. 1994, ApJ, 433, 303

Dyck, H. M., Benson, J. A., Ridgway, S. T., \& Dixon, D. J. 1992, AJ, 104, 1982 Freytag, B., Steffen, M., \& Dorch, B. 2002, Astron. Nachr., 323, 213

Gilliland, R. L., \& Dupree, A. K. 1996, ApJ, 463, L29

Goorvitch, D. 1994, ApJS, 95, 535

Gray, D. F. 2000, ApJ, 532, 487

Gray, D. F. 2008, AJ, 135, 1450

Haniff, C. 2007, New Astron. Rev., 51, 565

Harper, G. M., \& Brown, A. 2006, ApJ, 646, 1179

Haubois, X., Perrin, G., Lacour, S., et al. 2006, SF2A-2006, ed. D. Barret, F. Casoli, G. Lagache, A. Lecavelier, \& L. Pagani, 471

Hebden, J. C., Eckart, A., \& Keith Hege, E. 1987, ApJ, 314, 690

Hernández, O., \& Chelli, A. 2007, AMBER Scientific Workshop, presentation available at http://www-laog.obs.ujf-grenoble.fr/amber/IMG/ pdf/hernandez_Workshop_Q307LOAG.pdf

Hestroffer, D. 1997, A\&A, 327, 199

Hofmann, K.-H., Heininger, M., Jaffe, W., et al. 2008, SPIE Proc, 7013, 70133 Y

Huggins, P. J. 1987, ApJ, 313, 400

Huggins, P. J., Bachiller, R., Cox, P., \& Forveille, T. 1994, ApJ, 424, L127

Humphreys, R. M., Helton, L. A., \& Jones, T. 2007, AJ, 133, 2716

Jennings, D. E., Deming, D., Wiedemann, G. R., Keady, J. J. 1986, ApJ, 310, L39

Jennings, D. E., Sada, P. V. 1998, Science, 279, 844

Josselin, E., \& Plez, B. 2007, A\&A, 469, 671

Kervella, P., Coudé du Foresto, V., Glindemann, A., \& Hofmann, R. 2000, SPIE Proc., 4006, 31

Kervella, P., Ségransan, D., \& Coudé du Foresto, V. 2004, A\&A, 425, 1161

Kervella, P., Verhoelst, T., Ridgway, S. T., et al. 2009, A\&A, in press [arXiv: astro-ph/0907.1843]

Kiss, L. L., Szabó, Gy. M., \& Bedding, T. R. 2006, MNRAS, 372, 1721

Kraus, S., Weigelt, G., Balega, Y. Y., et al. 2009, A\&A, 497, 195

Lambert, D. L., Brown, J. A., Hinkle, K. H., \& Johnson, H. R. 1984, ApJ, 284, 223

Lim, J., Carilli, C., White, S. M., Beasley, A. J., \& Marson, R. G. 1998, Nature, 392,575
Lobel, A., \& Dupree, A. K. 2000, ApJ, 545, 454

Ludwig, H.-G., \& Beckers, J. 2008, ESO Astrophysics Symposia "The Power of Optical/IR Interferometry: Recent Scientific Results and 2nd Generation Instrumentation", ed. A. Richichi, F. Delplancke, F. Paresce, \& A. Chelli (Springer), 485

Meisner, J. A. 2003, Ap\&SS, 286, 119

Monnier, J. D., Tuthill, P. G., \& Danchi, W. C. 2000, ApJ, 545, 957

Ohnaka, K. 2004a, A\&A, 421, 1149

Ohnaka, K. 2004b, A\&A, 424, 1011

Perrin, G., Ridgway, S. T., Coudé du Foresto, V., et al. 2004, A\&A, 418, 675

Perrin, G., Ridgway, S. T., Verhoelst, T., et al. 2005, A\&A, 436, 317

Perrin, G., Verhoelst, T., Ridgway, S. T., et al. 2007, A\&A, 474, 599

Petrov, R. G., Malbet, F., Weigelt, G., et al. 2007, A\&A, 464, 1

Plez, B., \& Lambert, D. L. 2002, A\&A, 386, 1009

Richichi, A., \& Percheron, I. 2005, A\&A, 434, 1201

Ryde, N., Gustafsson, B., Hinkle, K. H., et al. 1999, A\&A, 347, L35

Ryde, N., Harper, G. M., Richter, M. J., Greathouse, T. K., \& Lacy, J. H. 2006a, ApJ, 637, 1040

Ryde, N., Richter, M. J., Harper, G. M., Eriksson, K., \& Lambert, D. L. 2006b, ApJ, 645, 652

Schröder, K.-P., \& Cuntz, M. 2005, ApJ, 630, L73

Schröder, K.-P., \& Cuntz, M. 2007, A\&A, 465, 593

Schwarzschild, M. 1975, ApJ, 195, 137

Smith, N., Hinkle, K. H., \& Ryde, N. 2009, AJ, 137, 3558

Smith, M., Patten, B. M., \& Goldberg, L. 1989, AJ, 98, 2233

Suzuki, T. K. 2007, ApJ, 659, 1592

Tatebe, K., Chandler, A. A., Wishnow, E. H., Hale, D. D. S., \& Townes, C. H. 2007, A\&A, 670, L21

Tatulli, E., Millour, F., Chelli, A., et al. 2007, A\&A, 464, 29

Townes, C. H., Wishnow, E. H., Hale, D. D. S., \& Walp, B. 2009, ApJ, 697, L127

Tsuji, T. 2000a, ApJ, 538, 801

Tsuji, T. 2000b, ApJ, 540, L99

Tsuji, T. 2006, ApJ, 645, 1448

Tsuji, T., Ohnaka, K., Hinkle, K. H., \& Ridgway, S. T. 1994, A\&A, 289, 469

Tuthill, P. G., Haniff, C. A., \& Baldwin, J. E. 1997, MNRAS, 285, 529

Uitenbroek, H., Dupree, A. K., \& Gilliland, R. L. 1998, ApJ, 116, 2501

Verhoelst, T., Decin, L., Van Malderen, R., et al. 2006, A\&A, 447, 311

Verhoelst, T., Van der Zypern, N., Hony, S., et al. 2009, A\&A, 498, 127

Vidotto, A. A., \& Janteco-Pereira, V. 2006, ApJ, 639, 416

Wallace, L., \& Hinkle, K. H. 1996, ApJS, 107, 312

Weigelt, G., Balega, Y. Y., Blöcker, T., et al. 2002, A\&A, 392, 131

Weigelt, G., Chesneau, O., Driebe, T., et al. 2008, SPIE Proc., 7013, 701303

Wittkowski, M., Aufdenberg, J. P., \& Kervella, P. 2004, A\&A, 413, 711

Woolf, N. J., Schwarzschild, M., \& Rose, W. K. 1964, ApJ, 140, 833

Young, J. S., Baldwin, J. E., Boysen, R. C., et al. 2000, MNRAS, 315, 635 